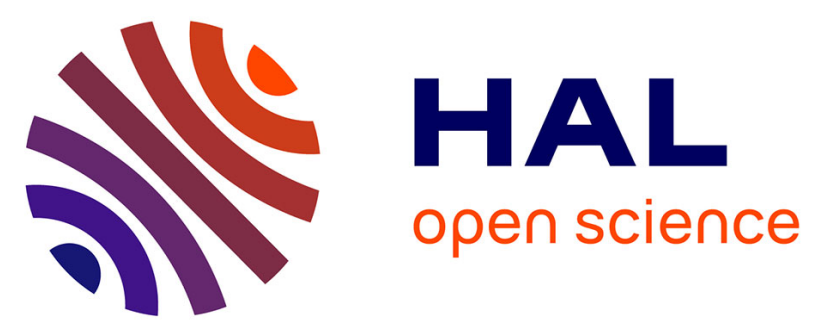

\title{
ÉVOLUTION POSTGLACIAIRE DES ENVIRONNEMENTS TRAVERTINEUX \\ PROVENÇAUX ET ALPINS : NOUVEAU CADRE CHRONOLOGIQUE, FACIĖS ET DYNAMIQUES MORPHOSÉDIMENTAIRES
}

Vincent Ollivier, Jean-Louis Guendon, Adam Ali, Paul Roiron, Paul Ambert

\section{To cite this version:}

Vincent Ollivier, Jean-Louis Guendon, Adam Ali, Paul Roiron, Paul Ambert. ÉVOLUTION POSTGLACIAIRE DES ENVIRONNEMENTS TRAVERTINEUX PROVENÇAUX ET ALPINS: NOUVEAU CADRE CHRONOLOGIQUE, FACIÈS ET DYNAMIQUES MORPHOSÉDIMENTAIRES. Quaternaire, 2006. hal-01766567

\section{HAL Id: hal-01766567 https://hal.science/hal-01766567}

Submitted on 25 May 2018

HAL is a multi-disciplinary open access archive for the deposit and dissemination of scientific research documents, whether they are published or not. The documents may come from teaching and research institutions in France or abroad, or from public or private research centers.
L'archive ouverte pluridisciplinaire HAL, est destinée au dépôt et à la diffusion de documents scientifiques de niveau recherche, publiés ou non, émanant des établissements d'enseignement et de recherche français ou étrangers, des laboratoires publics ou privés. 


\title{
ÉVOLUTION POSTGLACIAIRE DES ENVIRONNEMENTS TRAVERTINEUX PROVENÇAUX ET ALPINS : NOUVEAU CADRE CHRONOLOGIQUE, FACIÈS ET DYNAMIQUES MORPHOSÉDIMENTAIRES
}

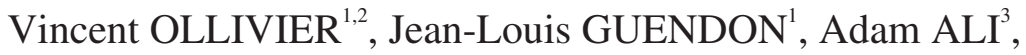 \\ Paul ROIRON ${ }^{3} \&$ Paul AMBERT $^{4}$
}

\begin{abstract}
RÉSUMÉ
Le début du développement des systèmes travertineux holocènes du sud de la France était auparavant rattaché à la période du Préboréal, et l'accumulation carbonatée principale à «l'optimum bioclimatique atlantique ». La «mort » progressive puis le démantèlement des formations s'effectuaient entre le Néolithique et la Pax romana. De nouvelles recherches démontrent un démarrage beaucoup plus précoce de la travertinisation dès le Tardiglaciaire. L'accumulation carbonatée perdure au Néolithique et ne s'interrompt réellement qu'au Petit Age Glaciaire. De nouvelles séquences plus complètes montrent l'importance du contexte géomorphologique dans la conservation des données stratigraphiques et leur représentativité. L'organisation des différents cycles sédimentaires au sein des formations travertineuses est précisée. Une définition des faciès carbonatés au regard des différentes caractéristiques des milieux de sédimentation est proposée. Des analyses paléoécologiques soulignent le caractère sensible des ensembles travertineux en fonction des modifications du biotope. Dès le Néolithique Final, dans un contexte d'augmentation des occupations humaines, les séquences enregistrent une série de ruptures. Ces perturbations sont accompagnées de changements de faciès et d'ouvertures fortes du milieu végétal mais ne modifient pas la tendance générale à la croissance des édifices. Ce n'est qu'entre le XIII ${ }^{\text {ème }}$ et le XVII ${ }^{\text {ème }}$ siècle après Jésus Christ que l'on note l'interruption de l'accumulation des travertins et le démantèlement des formations. C'est également dans le même intervalle que s'instaure la dynamique majeure d'incision linéaire des talwegs encore effective aujourd'hui.
\end{abstract}

Mots clés : Travertin, Tardiglaciaire, Holocène, paléoécologie, Néolithique, incision linéaire historique, sud-est de la France.

\section{ABSTRACT}

POSTGLACIAL EVOLUTION OF TRAVERTINE ENVIRONMENTS IN THE FRENCH ALPS AND PROVENCE : NEW CHRONOLOGY, FACIES, AND MORPHOSEDIMENTARY DYNAMICS

The beginning of the Holocene travertine system development in Southern France was formerly attributed to the Preboreal period and the main carbonated accumulation to the "bioclimatic atlantic optimum". The progressive decline and the dismantlement occurred between the Neolithic and the end of Roman times. New research has shown the beginning of the travertine system development in the Lateglacial period. The carbonated accumulation continued during the Neolithic and was only durably interrupted during the Little Ice Age. Newer complete stratigraphy has shown the importance of the geomorphological context in stratigraphical studies and their interpretation. The pattern of different sedimentary cycles within the travertinous formations became evident. Palaeo-ecological analyses undertaken have underlined the travertine sensitivity in accordance with biotope changes. Since the Final Neolithic, in the context of growing human occupation, ruptures in the travertine sequences have been recorded. These ruptures were accompanied by facies changing and strong vegetation opening in an ever growing travertine system. Between the XIIIth and the XVIIth century A.D., the decline and dismantlement of the travertine formations became noticeable. In the same way, the major final linear talweg incision was introduced in a morphogenic dynamic that is still happening today.

Key-words: Travertine, Lateglacial, Holocene, Palaeoecology, Neolithic, Historical linear incision, South-eastern France

\section{1 - INTRODUCTION}

Du Languedoc aux Alpes, le Sud de la France est un espace géographique où les formations travertineuses abondent (fig. 1). Variété des situations liée à la diversité des contextes paysagés, implication d'un large cadre chronologique (du Miocène à l'Holocène récent), relations privilégiées avec les occupations humaines, sensibilité aux multiples facteurs locaux de forte variabilité (évènements météorologiques, incendies,

${ }^{1}$ Economies Sociétés et Environnements Préhistoriques, UMR 6636, Maison Méditerranéenne des Sciences de l'Homme, BP 647, 5 rue du Château de l'Horloge, 13094 Aix en Provence Cedex 2, France. e-mail : vincent.ollivier@univ.u-3mrs.fr

${ }^{2}$ Institut Méditerranéen d'Ecologie et de Paléoécologie, UMR 6116, Bâtiment Villemin, Domaine du Petit Arbois, Avenue Philibert, BP 80 CEREGE, 13545 Aix en Provence Cedex 04, France.

${ }_{3}^{3}$ Centre de Bio-Archéologie et d'Ecologie, UMR 5059, Université de Montpellier 2, Institut de Botanique, 163 rue A. Broussonet, 34090 Montpellier, France.

${ }^{4}$ Centre d'Anthropologie, UMR 8555, 39 allées Jules-Guesde, 31080 Toulouse cedex, France. 


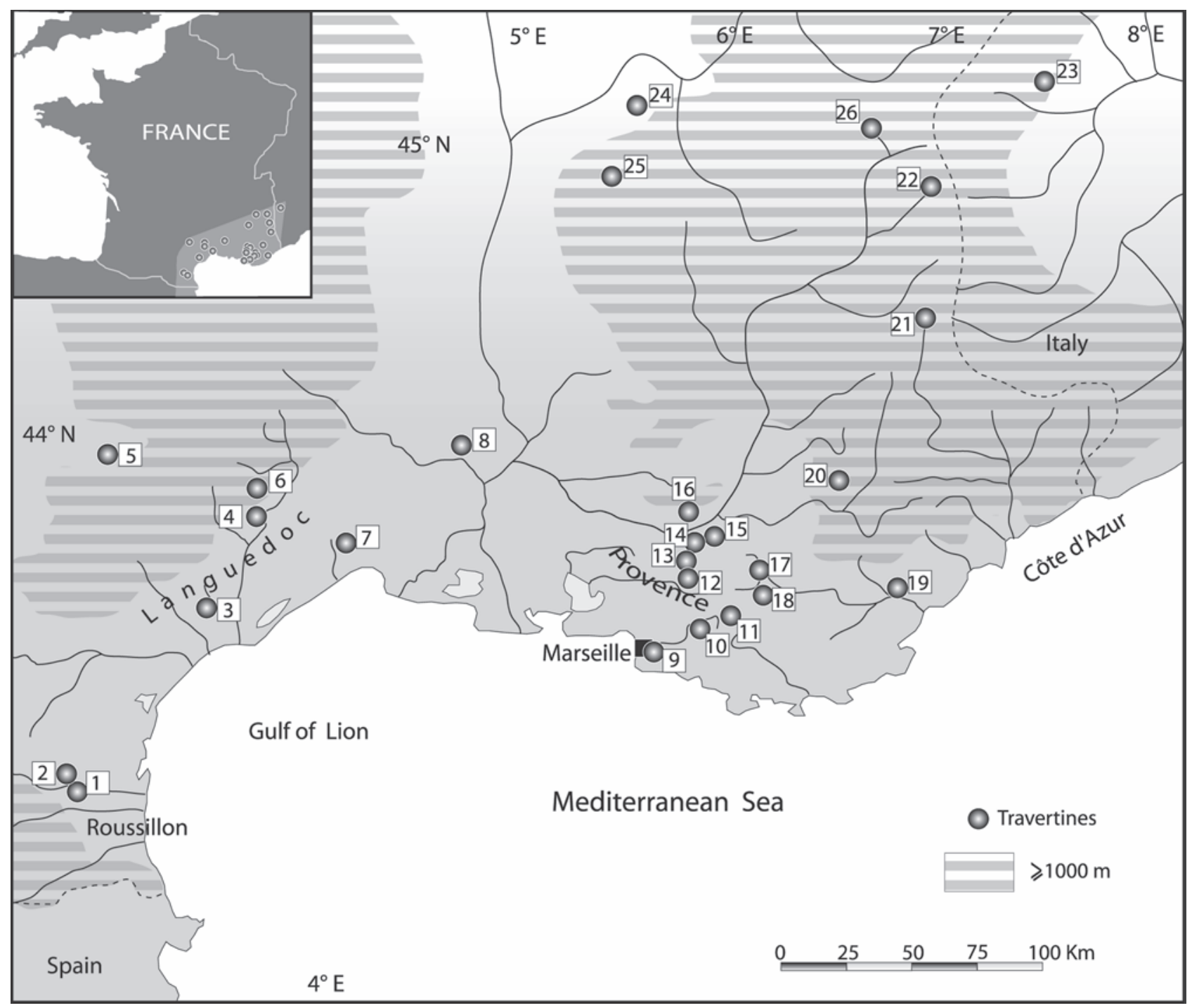

Fig. 1 : Localisation de quelques formations travertineuses postglaciaires emblématiques du sud de la France.

1) Maury ;2) Caramany ; 3) La Resclauze (Gabian) ; 4) St Guilhem-le-Désert ; 5) Millau ;6) Vallée de la Vis ; 7) Le Lez ; 8) Vallée de l'Alzon ; 9) Marseille (vallée de l'Huveaune) ; 10) Pont de Joux ; 11) St Baume ; 12) St Antonin ; 13) Vauvenargues ; 14) Meyrargues ; 15) Jouques ; 16) Luberon ; 17) Le Jonquier (Fontbrégoua) ; 18) Argens (Seillons) ; 19) Argens (La Motte) ; 20) Serre de Montdenier ; 21) St Etienne de Tinée ; 22) Queyras ; 23) Val de Susa (Italie) ; 24) Choranche (Vercors) ; 25) Omblèze (Vercors) ; 26) Col du Lautaret.

Fig. 1: Location of some emblematic Postglacial travertine formations of Southern France.

etc...) ou aux changements plus généraux (fluctuations climatiques globales, impact évolutif des sociétés humaines), sont les atouts majeurs des travertins du pourtour méditerranéen.

Carbonates externes construits d'origine biochimique et physico-chimique, les travertins ont fréquemment suscités un grand intérêt de la part des géologues, géographes, préhistoriens et paléoenvironnementalistes. Depuis le début des années 80 de véritables actions de recherche pluridisciplinaires, motivées par la richesse intrinsèque de ces formations, se sont développées du Languedoc à la Provence sous l'initiative de Jean Nicod (formations carbonatées externes tufs et travertins - Collectif 1981), Jean Vaudour (A.T.P. P.I.R.E.N. : Les édifices travertineux et l'histoire de l'environnement dans le midi de la France, - Vaudour 1988), Jean Louis Vernet et Jean Vaudour (A.T.P. P.I.R.E.N. Milieux et anthropisation à l'Holocène en Méditerranée occidentale à partir des sites karstiques, Vernet \& Vaudour, 1988-1990) ou encore Paul Ambert
(G.D.R. 1058 : Travertins et dépressions fermées de piémont: paléoenvironnements et anthropisation des paysages du midi méditerranéen, Ambert, 1997).

Les analyses actuelles, centrées sur la Provence et la zone alpine, s'inscrivent naturellement dans la continuité du travail réalisé par ces différentes actions scientifiques. Sont intégrés à ces nouvelles recherches les sites anciennement étudiés du col du Lautaret (Mlakar et al., 1999) dans les Hautes Alpes, de St Antonin (Guendon et Vaudour, 1981 ; Guendon et al., 2003) sur le versant sud de la montagne St Victoire (Bouches du Rhône), de même que Meyrargues (Bouches du Rhône, Magnin et al., 1991) en rive gauche de la Durance et Pont de Joux dans la vallée de l'Huveaune (D'Anna et al., 1988). D'autres formations, porteuses de données inédites et novatrices, s'ajoutent au corpus existant : le Queyras (Brotto, 1986; Ali et al., 2004), le Serre de Montdenier (Alpes de Haute-Provence, Nevière, 1996 ; Roiron et al., sous presse), et principalement les ensembles du sud Luberon (formations du 
Mirail, des Hermitans, et du Loup, communes de Peypin d'Aigues et de Cabrières d'Aigues, Vaucluse, Ollivier et al., 2004).

L'analyse multi-critères des séquences récemment découvertes entre moyenne Provence et domaine alpin permet de revisiter ces formations emblématiques. Les données sur les relations hommes/milieux travertineux se trouvent confortées, tandis que les limites chronologiques et les interrelations faciès/dynamiques morphosédimentaires et fluctuations paléohydrologiques, sont redéfinies à la lumière des nouvelles constatations.

\section{2 - OCCUPATIONS HUMAINES ET ENVIRONNEMENTS TRAVERTINEUX : DES RELATIONS PRIVILÉGIÉES}

Témoins essentiels de la transformation des géosystèmes naturels en géosystèmes anthropisés au cours du Postglaciaire (Vaudour, 1986a), les édifices travertineux contiennent de nombreux indices ou vestiges d'occupations humaines in situ ou dans leur environnement proche. Deux cas peuvent être distingués : celui des formations travertineuses fossiles et celui des systèmes travertineux actifs. Cette distinction est arbitraire car les deux cas peuvent coexister, les formations fossiles étant très souvent proches de systèmes encore actifs.

Parmi les formations travertineuses fossiles (aux époques des occupations concernées), les systèmes en balcon et en cascades présentent des caractères morphologiques qui en font des sites privilégiés : replats suspendus favorables à l'établissement de sites perchés et fortifiés, cavités naturelles recherchées comme lieux d'habitats en grottes, bergeries ou maisons et villages troglodytes. Les cas d'occupations préhistoriques semblent peu connus compte tenu des nombreuses, durables et importantes occupations plus « récentes » qui ont pu diluer l'information ou détériorer les vestiges antérieurs. Nous pouvons cependant évoquer l'habitat magdalénien du site de l'Estabel à Cabrières dans l'Hérault (Ambert, 1979-1981 ; 1993-1998), ou encore les multiples occupations du site de Ségriès (travertins du Pléistocène inférieur) qui s'échelonnent du Moustérien à l'époque contemporaine (Dubar \& Clappier, 1989) et de l'abris du Rouet (Carry-Le-Rouet) en ce qui concerne le Tardigravettien (Brochier, communication orale ; Brochier \& Livache, 2003).

L'abondance, la pérennité et la qualité des eaux sont sans doute les facteurs essentiels qui font des systèmes travertineux actifs, qu'ils soient alluviaux, de sources, cascadants ou palustres, des sites attractifs fréquentés et occupés de tous temps par les populations humaines. Sans exhaustivité, les travertins de Vendres (Ambert, 1982, 1991) ou de Millau, la Rouquette (Ambert et al., 1989), sont de bons exemples pour le Paléolithique inférieur et moyen, tout comme Pont de Joux en Provence (racloir double moustérien, Paléolithique moyen, D’Anna et al., 1988).

Avec les périodes plus récentes du Néolithique les occupations de plein air en milieux travertineux deviennent plus marquées et les interactions sur la sédimentation et l'environnement sont alors plus sensibles. Les cas sont multiples et quasi généralisés de la Provence au Languedoc au moins dès le Néolithique moyen (D'Anna \& Courtin, 1986). A titre d'exemples nous pouvons citer les formations de : St Antonin où l'avant dernier ensemble sédimentaire daté à $4840 \pm$ $80 \mathrm{BP}$ intègre du mobilier archéologique provenant de gisements périphériques attribués au Chasséen/Néolithique final, (Guendon et al., 2003) ; Vauvenargues (gisement Néolithique final/Chalcolithique en place vers 3040 BC, Cheylan \& D'Anna, 1979 ; D’Anna \& Courtin, 1986) ; du Mirail et des Hermitans (niveaux d'occupations du Néolithique final entre $4737 \pm 47 \mathrm{BP}$ et $3396 \pm 30 \mathrm{BP}$, Ollivier et al., 2004) ou de la Resclauze à Gabian (Ambert \& Delgiovine, 1979 ; Ambert, 1988 ; Brochier, 1988).

Bien que souvent bref (Brochier, 1988, 2002), l'impact des modes d'occupation humaine au Néolithique semble avoir une influence sur le bilan accumulation/érosion dans le développement des séquences travertineuses. Dans ce cas précis, une conjonction de facteurs en rétroaction positive, tel que variabilité climatique intra-holocène + poids des occupations humaines ou sensibilité acquise des milieux travertineux + poids des occupations humaines, semble nécessaire pour que les effets de ces «anthropisations » soient plus nettement marqués (cas du Mirail, Ollivier et al., 2006, de St Antonin, Guendon et al., 2003).

\section{3 - NOUVELLE CHRONOLOGIE DE LA TRAVERTINISATION POSTGLACIAIRE}

\section{1 - DES ACCUMULATIONS TRAVERTINEUSES PRÉCOCES}

Malgré la diversité des dates postglaciaires (du Préboréal à la fin de l'Atlantique) préalablement obtenues (Vaudour, 1986 et fig. 2), le démarrage de la travertinisation dans le sud de la France était considéré comme inféodé à l'optimum bioclimatique atlantique (aux alentours de 7500 BP). De nombreuses formations avaient effectivement délivré des datations centrées sur cette période (cas de Jouques, St Paul les Durance, Meyrargues, Pont de Joux, Serre de Montdenier-bassin en Provence, de la Vis, du Lez, de St Guilhem-leDésert et de la Resclauze en Languedoc) définie alors comme chronozone de prédilection du développement optimal des édifices carbonatés (Vaudour, 1994). Au fur et à mesure de l'avancée des recherches, de nouvelles datations ${ }^{14} \mathrm{C}$ et $\mathrm{U} / \mathrm{Th}$ ont fréquemment démontré l'existence d'accumulations carbonatées plus précoces débutant dès le Préboréal ou à la transition PréboréalBoréal (fig. 2), aussi bien en domaine méditerranéen à méso-méditerranéen (St Antonin, Vauvenargues, La Tave; Guendon et al., 2003 ; Cheylan et D'Anna, 1979 ; Magnin, 1997), que dans les contextes environnementaux plus sensibles de l'étage supra-méditerranéen (Serre de Montdenier-amont, Omblèze, Roiron, 


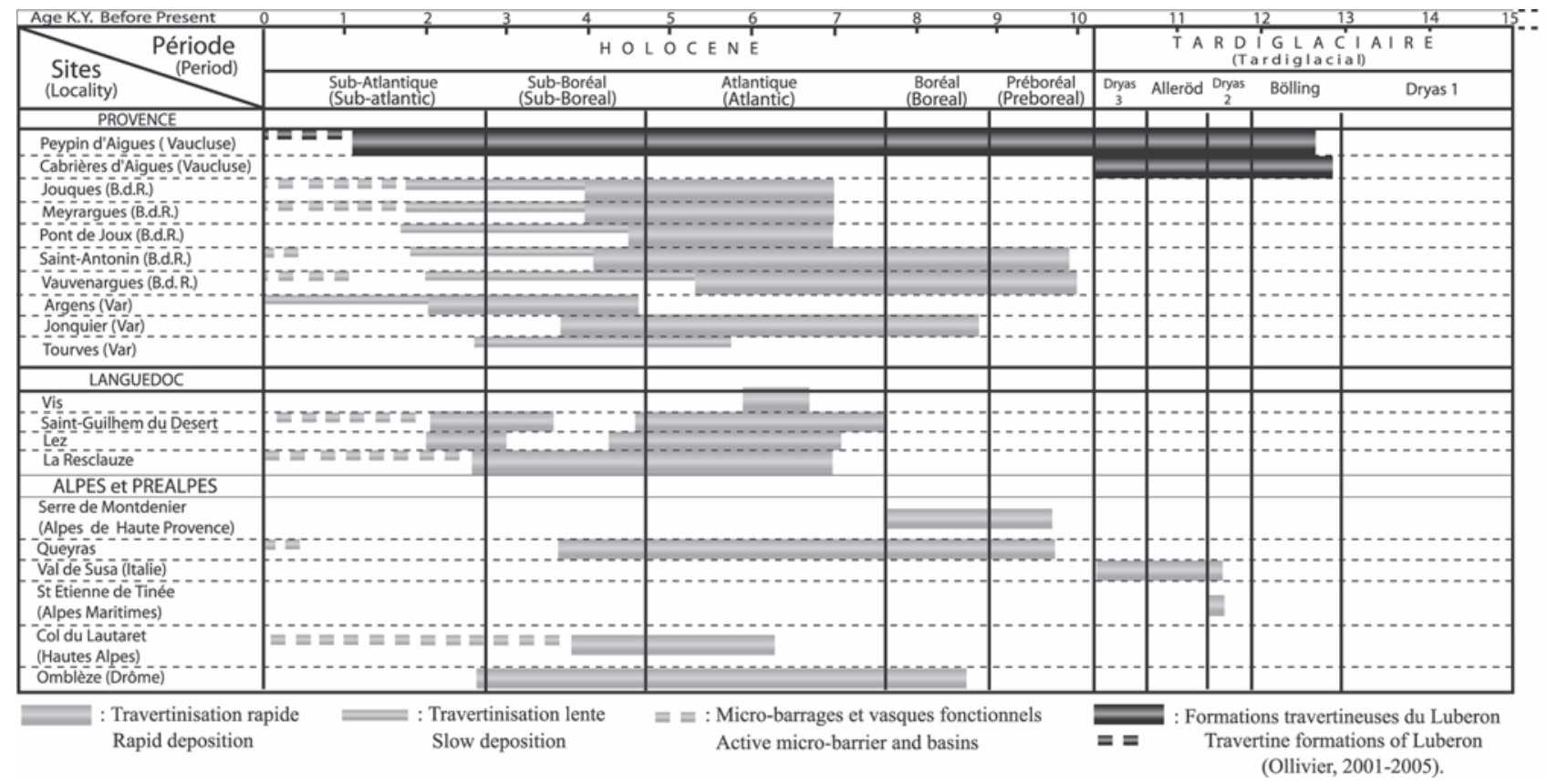

Fig. 2 : Synthèse chronologique des principales formations travertineuses postglaciaires du sud de la France.

Fig. 2: Chronological synthesis of the main Postglacial travertine formations of Southern France.

ce numéro, Diaz Del Olmo et al., 1997) à alpin d'altitude (Les Tioures, Queyras, Ali et al., 2004).

Sur le piémont méridional du Grand Luberon, l'accumulation des travertins a fonctionné durant le Tardiglaciaire (Bølling), période à l'ambiance climatique encore fraîche (GS2a, Greenland stadial a, INTIMATE groupe, Bjorck et al., 1998 ; Walker et al., 1999 ; Lowe et al., 2001, et fig. 2). Ces nouvelles données constituent une originalité dans le cadre de l'étude des systèmes et des paléoenvironnements travertineux postglaciaires.

Les séquences travertineuses des ravins du Loup, du Mirail, et des Hermitans, sont localisées à l'aval de petite exsurgences karstiques développées au niveau des calcaires et marno-calcaires valanginiens qui constituent le cœur de l'anticlinal du Grand Luberon (Ollivier et al., 2004). D'une puissance moyenne avoisinant la douzaine de mètres, ces formations se sont exprimées depuis le Tardiglaciaire sous une forme crayeuse dès $12900 \pm 60 \mathrm{BP} / 12640 \pm 60 \mathrm{BP}$ dans les vallons du Loup et du Mirail puis, postérieurement à $10390 \pm 50 \mathrm{BP}$ aux Hermitans (fig. 3 et tab. 1). Seuls quelques niveaux détritiques (cailloutis calcaires hétérométriques et subémoussés liés à des épisodes de crues ou à une mobilité latérale du chenal principal) ou des sols hydromorphes viennent ponctuer l'ensemble des dépôts sans toutefois perturber ou inhiber l'accumulation travertineuse.

Le caractère précoce du début de l'accumulation carbonatée, par comparaison avec les données antérieures (Vaudour, 1986, 1994), ne semble pas être une spécificité des séquences du Luberon. D’autres formations plus lointaines démontrent aussi un démarrage tardiglaciaire de l'accumulation de travertins (fig. 2) notamment au niveau de St Etienne de Tinée (datation ${ }^{14} \mathrm{C}$ à $11460 \pm 50$ BP, Bigot-Cormier et al., 2005), de San Dominico (commune d'Oulx, val de Susa, Italie) sur le versant est du Mont Genèvre (U/Th sur bloc de travertin soliflué à $11506 \pm 66 \mathrm{BP}$, Martinez, 2005) et dans la même vallée, de la formation de La Selle datée en U/Th à $10145 \pm 225$ BP (Roiron, communication orale).

L'ensemble de ces résultats souligne la complexité des liens établis entre le développement des systèmes travertineux et l'ambiance climatique et biologique qui leur sont associé. Même si ces formations restent statistiquement, tant du point de vue de leur volume que de leur répartition, plus nombreuses et mieux développées en milieux tempérés, divers travaux ont signalé la présence de travertins dans des domaines montagnards d'altitude, au climat froid et rigoureux. On peut citer par exemple : le Queyras (Ali et al., 2004), le col du Lautaret (Mlakar, 1999), ou dans des contrées plus lointaines, l'Afghanistan (Lang \& Lucas, 1970) l'Himalaya (Freytet et Fort, 1980 ; Fort, 1981) ou encore l'Argentine (Valero-Garcés et al., 2001). Le chimisme des eaux lié au degré d'évolution du karst apparaît aussi comme un élément déterminant dans le cadre du développement de ces formations (Magnin et al., 1991). Il convient donc de distinguer le potentiel de travertinisation des eaux (généralement peu variable au cours de l'Holocène) de celui de la capacité d'accumulation des systèmes carbonatés, variable fortement dépendante des fluctuations paléohydrologiques et du rapport: intensité du dépôt du $\mathrm{CaCO}_{3} /$ intensité de l'érosion (Bakalowicz, 1988).

\section{$3.2-\mathrm{UN}$ «DÉCLIN » RAPIDE ET TARDIF}

Dans une même optique, l'ensemble des travaux précédemment réalisés sur les travertins du Sud de la France indiquait un « déclin » puis un démantèlement effectif des formations carbonatées aux alentours du Néolithique voir, dans certains cas, de l'Antiquité 


\begin{tabular}{|c|c|c|c|c|c|c|}
\hline $\begin{array}{c}\mathrm{N}^{\circ} \\
\text { laboratoire } \\
\text { (Laboratory } \\
n^{\circ} \text { ) }\end{array}$ & $\begin{array}{c}\text { Datation }{ }^{14} \mathrm{C} \\
\text { uncal. BP }\end{array}$ & $\begin{array}{c}\text { Datation }{ }^{14} \mathrm{C} \\
\text { cal. BP }\end{array}$ & $\begin{array}{c}\text { Datation }{ }^{14} \mathrm{C} \text { cal. } \\
\mathrm{BC} / \mathrm{AD}\end{array}$ & $\begin{array}{l}\text { Support et } \\
\text { méthode } \\
\text { (material and } \\
\text { method) }\end{array}$ & $\begin{array}{l}\text { Ravin } \\
\text { concerné } \\
\text { (site) }\end{array}$ & $\begin{array}{l}\text { Commune } \\
\text { (Locality) }\end{array}$ \\
\hline Poz-7792 & $\begin{array}{c}12900 \pm 60 \\
B P\end{array}$ & $\begin{array}{c}15531-14981 \\
\text { cal BP }\end{array}$ & $\begin{array}{c}13581-13031 \\
\text { cal BC }\end{array}$ & $\begin{array}{l}\text { Bois fossile (wood) } \\
\text { AMS }\end{array}$ & Loup & Cabrières d'Aigues \\
\hline Poz-7825 & $\begin{array}{c}12640 \pm 60 \\
B P\end{array}$ & $\begin{array}{c}15190-14610 \\
\text { cal BP }\end{array}$ & $\begin{array}{c}13240-12660 \\
\text { cal BC }\end{array}$ & $\begin{array}{c}\text { Charbon (charcoal) } \\
\text { AMS }\end{array}$ & Mirail & Peypin d'Aigues \\
\hline Poz-12381 & $\begin{array}{c}12110 \pm 60 \\
\mathrm{BP} \\
\end{array}$ & $\begin{array}{c}14107-13814 \text { cal } \\
\text { BP }\end{array}$ & $\begin{array}{c}\text { 12157- 11864 cal } \\
\text { BC }\end{array}$ & $\begin{array}{c}\text { Charbon (charcoal) } \\
\text { AMS }\end{array}$ & Loup & Cabrières d'Aigues \\
\hline Poz-7790 & $\begin{array}{c}11650 \pm 60 \\
\mathrm{BP}\end{array}$ & $\begin{array}{c}13669-13348 \\
\text { cal BP }\end{array}$ & $\begin{array}{c}11719-11398 \\
\text { cal BC }\end{array}$ & $\begin{array}{c}\text { Charbon (charcoal) } \\
\text { AMS }\end{array}$ & Loup & Cabrières d'Aigues \\
\hline Poz-12440 & $\begin{array}{c}11620 \pm 60 \\
\mathrm{BP}\end{array}$ & $\begin{array}{c}\text { 13638- } 13318 \\
\text { cal BP }\end{array}$ & $\begin{array}{c}11688-11368 \mathrm{cal} \\
\mathrm{BC}\end{array}$ & $\begin{array}{c}\text { Charbon (charcoal) } \\
\text { AMS } \\
\end{array}$ & Mirail & Peypin d'Aigues \\
\hline Poz-12428 & $\begin{array}{c}10390 \pm 50 \\
\mathrm{BP}\end{array}$ & $\begin{array}{c}\text { 12401- 12067 } \\
\text { cal BP }\end{array}$ & $\begin{array}{c}\text { 10451- 10117 cal } \\
\text { BC }\end{array}$ & $\begin{array}{c}\text { Charbon (charcoal) } \\
\text { AMS } \\
\end{array}$ & Hermi & Peypin \\
\hline Poz-12382 & $\begin{array}{c}10210 \pm 50 \\
\mathrm{BP}\end{array}$ & $\begin{array}{c}\text { 12104- } 11746 \\
\text { cal BP }\end{array}$ & $\begin{array}{c}10154-9796 \mathrm{cal} \\
\text { BC } \\
\end{array}$ & $\begin{array}{c}\text { Charbon (charcoal) } \\
\text { AMS }\end{array}$ & Loup & 'Aigues \\
\hline Lyon 10522 & $7775 \pm 65 \mathrm{BP}$ & $\begin{array}{c}8718-8414 \text { cal } \\
\text { BP }\end{array}$ & $\begin{array}{c}\text { 6768- } 6464 \text { cal } \\
\text { BC } \\
\end{array}$ & $\begin{array}{l}\text { Charbon standard } \\
\text { (charcoal standard) }\end{array}$ & Mirail & gues \\
\hline Poz-7802 & BP & $\begin{array}{c}8356-8156 \mathrm{cal} \\
\text { BP }\end{array}$ & $\begin{array}{c}\text { 6406- } 6206 \mathrm{cal} \\
\mathrm{BC} \\
\end{array}$ & $\begin{array}{l}\text { rbon (charcoal) } \\
\text { AMS }\end{array}$ & $\mathrm{Mi}$ & Peyl \\
\hline Poz-7794 & $330 \pm 40 \mathrm{BP}$ & \begin{tabular}{|c|}
$7739-7588$ cal \\
BP \\
\end{tabular} & $\begin{array}{c}5789-5638 \text { cal } \\
\text { BC } \\
\end{array}$ & $\begin{array}{r}\text { Charbon ( } \\
\text { AN }\end{array}$ & Mirail & Aigues \\
\hline Lyon 10574 & $550 \pm 95 \mathrm{BP}$ & $\begin{array}{c}7587-7275 \text { cal } \\
\text { BP } \\
\end{array}$ & $\begin{array}{c}5637-5325 \text { cal } \\
\text { BC } \\
\end{array}$ & $\begin{array}{l}\text { Charbon standard } \\
\text { (charcoal standard) }\end{array}$ & Mirail & ues \\
\hline Poz & $\mathrm{P}$ & $\begin{array}{c}7477-7318 \text { cal } \\
\text { BP }\end{array}$ & $\begin{array}{c}5527-5368 \text { cal } \\
\text { BC } \\
\end{array}$ & $\begin{array}{r}\text { Charbon (ch } \\
\text { AMS }\end{array}$ & Mirail & $\mathrm{Pe}$ \\
\hline Poz-7826 & $20 \pm 35 \mathrm{BP}$ & $\begin{array}{c}6573-6436 \mathrm{cal} \\
\text { BP } \\
\end{array}$ & $\begin{array}{c}4623-4486 \mathrm{cal} \\
\mathrm{BC} \\
\end{array}$ & $\begin{array}{r}\text { Charbon } \\
\text { AN } \\
\end{array}$ & Mirail & ues \\
\hline Poz-12426 & $20 \pm 40 \mathrm{BP}$ & $\begin{array}{c}\text { 6403- 6277 cal } \\
\text { BP }\end{array}$ & $\begin{array}{c}\text { 4453- } 4327 \text { cal } \\
\text { BC } \\
\end{array}$ & $\begin{array}{r}\text { Charbon ( } \\
\mathrm{AN}\end{array}$ & Her & $\mathrm{Pe}$ \\
\hline$\overline{\mathrm{AA} 4}$ & BP & $\begin{array}{c}5587-5446 \text { cal } \\
\text { BP } \\
\end{array}$ & $\begin{array}{c}\text { 3637- } 3496 \mathrm{cal} \\
\text { BC } \\
\end{array}$ & $\begin{array}{c}\text { Charbon standard } \\
\text { (charcoal standard) }\end{array}$ & 10 & $\mathrm{Pe}$ \\
\hline AA42670 & $36 \pm 56 \mathrm{BP}$ & $\begin{array}{c}5323-5036 \mathrm{cal} \\
\mathrm{BP} \\
\end{array}$ & $\begin{array}{c}\text { 3373-3086 cal } \\
\text { BC } \\
\end{array}$ & $\begin{array}{l}\text { Charbon standard } \\
\text { (charcoal standard) }\end{array}$ & Mirail & \\
\hline Poz-7904 & $340 \pm 40 \mathrm{BP}$ & $\begin{array}{c}4979-4840 \mathrm{cal} \\
\mathrm{BP} \\
\end{array}$ & $\begin{array}{c}\text { 3029-2890 cal } \\
\text { BC }\end{array}$ & $\begin{array}{c}\text { Charbon (charcoal) } \\
\text { AMS } \\
\end{array}$ & Hern & Pey \\
\hline Poz-7793 & $5 \mathrm{BP}$ & $\begin{array}{c}4533-4410 \mathrm{cal} \\
\text { BP }\end{array}$ & $\begin{array}{c}2585-2460 \text { cal } \\
\text { BC }\end{array}$ & $\begin{array}{r}\text { Charbon } \\
\mathrm{A} \\
\end{array}$ & 10 & Pey \\
\hline Poz-7796 & $90 \pm 35 \mathrm{BP}$ & $\begin{array}{c}\text { 4293- } 4082 \text { cal } \\
\text { BP }\end{array}$ & $\begin{array}{c}\text { 2343-2132 cal } \\
\text { BC } \\
\end{array}$ & $\begin{array}{r}\text { Charbon } \\
\mathrm{AI} \\
\end{array}$ & Mirail & ues \\
\hline Poz-7801 & BP & $\begin{array}{c}3709-3566 \mathrm{cal} \\
\text { BP } \\
\end{array}$ & $\begin{array}{c}1759-1616 \mathrm{cal} \\
\text { BC }\end{array}$ & $\begin{array}{c}\text { Charbon (charcoal) } \\
\text { AMS }\end{array}$ & $\mathrm{Her}$ & Pey \\
\hline Poz-7827 & $00 \pm 30 \mathrm{BP}$ & $\begin{array}{c}1575-1758 \mathrm{cal} \\
\text { BP } \\
\end{array}$ & 375- 192 cal BC & $\begin{array}{r}\text { Charbon } \\
\mathrm{A} \\
\end{array}$ & MHall & Peypin \\
\hline Poz-7823 & $80 \pm 30 \mathrm{BP}$ & $\begin{array}{c}1884-1728 \text { cal } \\
\text { BP }\end{array}$ & 66- 222 cal AD & $\begin{array}{c}\text { Charbon (charcoal) } \\
\text { AMS } \\
\end{array}$ & Mirail & igues \\
\hline Poz-7798 & $\mathrm{BP}$ & \begin{tabular}{|c|}
$1819-1689$ cal \\
BP
\end{tabular} & 131- 261 cal AD & $\begin{array}{c}\text { Charbon (charcoal) } \\
\text { AMS } \\
\end{array}$ & Mirail & es \\
\hline Poz-7803 & $1775 \pm 30 \mathrm{BP}$ & $\begin{array}{c}\text { 1745-1610 cal } \\
\text { BP } \\
\end{array}$ & 205- 340 cal AD & $\begin{array}{r}\text { Charbon } \\
\mathrm{A}\end{array}$ & Mirail & Peypin \\
\hline Poz-12439 & $1385 \pm 30 \mathrm{BP}$ & $\begin{array}{c}\text { 1345- } 1275 \text { cal } \\
\text { BP }\end{array}$ & $605-675$ cal AD & $\begin{array}{c}\text { Charbon (charcoal) } \\
\text { AMS }\end{array}$ & Hermitans & Peypin d'Aigues \\
\hline Poz-7799 & $1210 \pm 30 \mathrm{BP}$ & $\begin{array}{c}1185-1059 \text { cal } \\
\text { BP }\end{array}$ & 765-891 cal AD & $\begin{array}{c}\text { Charbon (charcoal) } \\
\text { AMS } \\
\end{array}$ & Mirail & Peypin d'Aigues \\
\hline Poz-12386 & $1145 \pm 30 \mathrm{BP}$ & $\begin{array}{c}1144-973 \text { cal } \\
\text { BP }\end{array}$ & 806- 977 cal AD & $\begin{array}{c}\text { Charbon (charcoal) } \\
\text { AMS }\end{array}$ & Loup & Cabrières d'Aigues \\
\hline Poz-7903 & $1080 \pm 30 \mathrm{BP}$ & $\begin{array}{c}\text { 1016- } 933 \text { cal } \\
\text { BP } \\
\end{array}$ & $\begin{array}{c}\text { 934- 1017 cal } \\
\text { AD } \\
\end{array}$ & $\begin{array}{c}\text { Charbon (charcoal) } \\
\text { AMS } \\
\end{array}$ & Mirail & Peypin d'Aigues \\
\hline Poz-12435 & $780 \pm 30 \mathrm{BP}$ & $737-670$ cal BP & $\begin{array}{c}1213-1280 \mathrm{cal} \\
\mathrm{AD}\end{array}$ & $\begin{array}{c}\text { Charbon (charcoal) } \\
\text { AMS }\end{array}$ & Mirail & Peypin d'Aigues \\
\hline $\mathrm{Poz}$ & $325=$ & 470-306 cal BP & $\begin{array}{c}1480-1644 \text { cal } \\
\text { AD }\end{array}$ & $\begin{array}{c}\text { Charbon (charcoal) } \\
\text { AMS } \\
\end{array}$ & Loup & $\mathrm{Cal}$ \\
\hline
\end{tabular}

Tab. 1 : Récapitulatif des datations ${ }^{14} \mathrm{C}$ (A.M.S.) obtenues dans les formations travertineuses postglaciaire du Luberon (Vaucluse) (calibration : Calib. 5, Radiocarbon Calibration Program, Copyright 1986-2005 M Stuiver \& PJ Reimer).

Tab. 1: Synthesis of the radiocarbon ${ }^{14}$ C (A.M.S.) analysis of the Postglacial travertine formation of the Luberon, Vaucluse). 

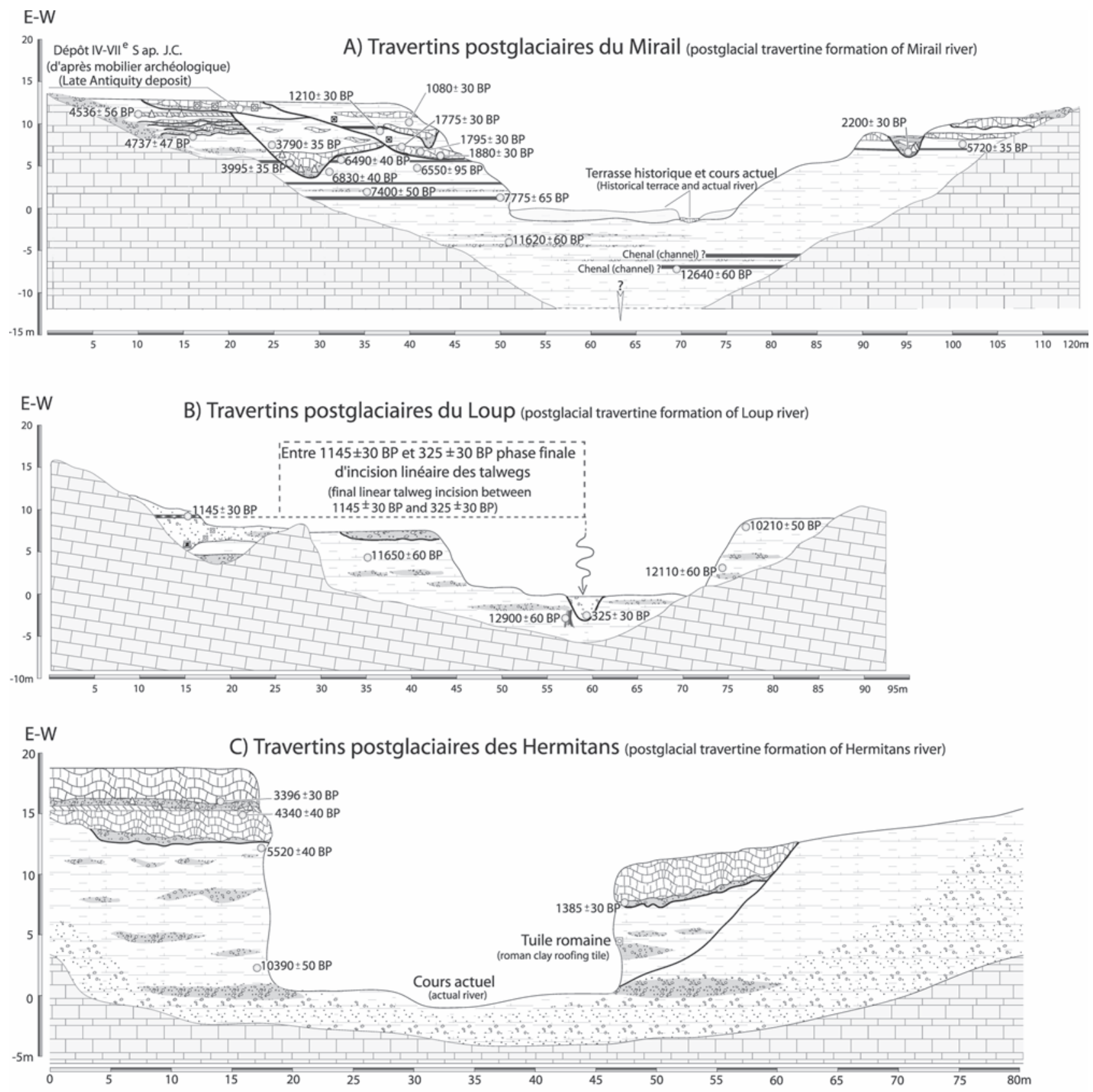

LEGENDE (LEGEN)

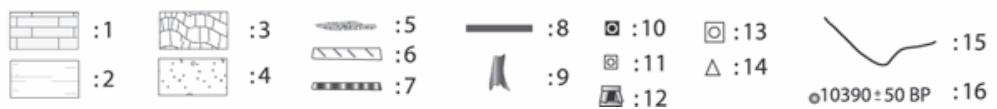

Fig. 3 : Profils synthétiques des formations travertineuses postglaciaires du Luberon (Vaucluse).

Legende : 1) Substratum paléogène et néogène (calcaires, marno-calcaires, argiles, molasses) ;2) Craies travertineuses ; 3) Travertin construit ;4) Limons sables et cailloutis ; 5) Nappe caillouteuse ; 6) Niveau d'occupation humaine du Néolithique final ; 7) Esquisse de sol ; 8) Sol hydromorphe ; 9) Tronc sub-fossile de type Pinus Sylvestris ; 10) Tesson Antiquité Tardive à Moyen Age ; 11) Fragment de tuile antique ou Antiquité Tardive ; 12) Conduite antique ou Antiquité Tardive ; 13) Tuile romaine ; 14) Tesson Néolithique final ; 15) Incision ; 16).Datation ${ }^{14} \mathrm{C}$.

Fig. 3: Synthetic transverse profile of Postglacial travertine formations of Luberon, Vaucluse.

Legend : 1) Paleogenic and neogenic substrate ;2) Chalk-travertine ;3) Travertine ;4) Silts, sands and pebbles ; 5) Pebbles and gravel ;6) Human occupation level, Late Neolithic ; 7) Soil ; 8) Hydromorphic soil ; 9) Sub-fossil trunck ; 10) Ceramic of Late Antiquity to Middle Age ; 11) Antiquity or Late Antiquity clay roofing tile ; 12) Antiquity or Late Antiquity pipe flow ; 13) Roman clay roofing tile ; 14) Late Neolithic ceramic ; 15) Incision ; 16) ${ }^{14} \mathrm{C}$ datation. 
(Vaudour, 1986, 1994). Si une somme importante de datations souligne ou confirme cette hypothèse pour une grande partie des systèmes travertineux méditerranéens (Vaudour, 1994 ; Magnin et Thinon, 1988 ; Magnin et al., 1991; Cheylan \& D’Anna, 1979; D'Anna et Courtin, 1986; Guendon et al., 2003 ; Ambert \& Gasco, 1989 ; Ambert \& Delgiovine, 1979 ; Nicol-Pichard, 1986, etc.) de nouvelles données obtenues dans le Luberon rajeunissent le démantèlement puis l'interruption (ou le ralentissement majeur), de l'accumulation carbonatée postglaciaire en Provence (fig. 2 et tab. 1).

Après un développement visiblement continu d'environ $13000 \mathrm{BP}$ à $5500 \mathrm{BP}$ (fig. 3), uniquement ponctué à l'Atlantique par quelques passées détritiques et des sols hydromorphes, les formations du Luberon enregistrent une série de perturbations de haute fréquence et de faible amplitude dès $4737 \pm 47 \mathrm{BP}$. Ces perturbations, qui en fonction de leur importance seront qualifiées de ruptures, se multiplient du Néolithique final à l'Epoque historique en prenant la forme d'incisions brèves et rapides (d'ampleur variable d'amont en aval) dans les dépôts sous-jacents sans modification notable du bilan global de l'accumulation travertineuse qui perdure au-delà de $1080 \pm 30 \mathrm{BP}$ (fig. 2, fig. 3 et tab. 1). Ces fluctuations morphosédimentaires (accumulation/incision) sont généralement suivies de changements radicaux de faciès dont nous discuterons l'aspect et la signification.

Le déclin, ou plutôt la rupture dans la dynamique d'accumulation des séquences travertineuses (car celle-ci perdure aujourd' hui, sans pour autant atteindre les volumes ou les surfaces passées, Baker \& Sims, 1998), semble étroitement et intimement liée au renversement de tendance en faveur de l'incision linéaire des talwegs (localement remarquable par sa rapidité, Gautier, 1992 ; Ollivier \& Pinatel, 2000). Cette dernière affecte les formations détritiques des Alpes à la Provence dans la seconde moitié de l'Holocène (Jorda et al., 2002 ; Bruneton et al., 2002 ; Ollivier et al., 2004). Dans le Luberon (Ollivier, thèse en cours), il apparaît clairement que cette dynamique d'incision, qui s'exprime encore largement aujourd'hui indépendamment de l'origine des dépôts (travertineux ou non), s'est amorcée en plusieurs étapes dès le $X^{\text {ème }}$ siècle (partie supérieure des coupes à $1145 \pm 30 \mathrm{BP}$ et $1080 \pm 30 \mathrm{BP}$, fig. 3 et tableau 1). Les dernières datations ${ }^{14} \mathrm{C}$ réalisées sur un large corpus amont-aval de formations détritiques et de séquences travertineuses réparties sur plusieurs ravins, indiquent que la phase terminale d'incision linéaire des talwegs se déroule précisément dans la période comprise entre le XIII ${ }^{\text {ème }}$ (sommet de la plupart des coupes stratigraphiques étudiées) et le XVII ${ }^{\text {ème }}$ siècle (datation de la nappe alluviale de fond de vallon à $325 \pm 30 \mathrm{BP}$, fig. 3 et tab. 1).

Le «démantèlement des édifices travertineux et le creusement des vallées » ne s'effectueraient donc pas entre la «Pax romana et le Moyen Age » (Vaudour, 1994) mais au Petit Age Glaciaire comme le montreraient les résultats obtenus dans le Luberon. Les modalités précises de la mise en place de cette incision restent toutefois à déterminer: poids des pressions liées aux modes d'occupation et aux activités humaines exercées sur l'environnement additionné au « coup de froid » climatique ?

\section{3 - UNE LECTURE DIACHRONIQUE DU DÉVELOPPEMENT DES SYSTÈMES TRAVERTINEUX DU SUD DE LA FRANCE LIÉE AUX CARAC- TÉRISTIQUES GÉOMORPHOLOGIQUES DES SITES ?}

Nous l'avons vu, de nombreuses coupes stratigraphiques dans les systèmes travertineux du Sud de la France ont été datées à la base aux environs de 7000 BP (fig. 2). Cette constatation renvoyait l'image d'un développement préférentiel des formations travertineuses durant l'optimum bioclimatique atlantique et non dès la première partie de l'Holocène, comme l'ont démontré les données ultérieures. Ces datations atlantiques pourraient relever d'un artefact inhérent à la morphologie de certaines vallées et de l'impact des multiples variations paléohydrologiques intra-holocènes sur les différentes générations de dépôts.

La position proximale ou distale de la séquence stratigraphique étudiée par rapport à l'axe du talweg semble être un élément déterminant (Ollivier et al., 2006), notamment dans le cas des édifices travertineux, systèmes au fort développement vertical favorisant à terme la transgression latérale des dépôts sur les bordures. Les complexes sédimentaires qui se développent dans de « larges » vallées, sur des pentes douces ou sur des piémonts au drainage irrégulier (Luberon) sont généralement bien conservés, autorisant une lecture séquentielle relativement complète intégrant le chenal principal et ses marges. En revanche, dans le cas de vallons étroits favorisant la concentration des écoulements et limitant l'étendue des formations, ces derniers peuvent être recoupés dans le temps par l'organisme qui en est à l'origine. Il ne reste en héritage que les séquences de bordures amputées de leurs premiers dépôts qui étaient uniquement présents dans l'axe du talweg. L'intégration des sites étudiés dans leur contexte géomorphologique est donc un élément de première importance pour une bonne compréhension de la signification des séquences stratigraphiques et de leur représentativité.

Plusieurs formations travertineuses provençales peuvent conforter ce point de vue :

- A Meyrargues (fig. 4) la seule coupe qui subsiste des travertins holocènes repose latéralement à l'axe du paléotalweg. La séquence complète centrée sur l'axe du chenal principal a probablement été évacuée par de multiples incisions. L'information reflétée se trouve donc incomplète, l'accumulation carbonatée est diagnostiquée comme débutant à l'Atlantique (7060 \pm 200 BP, fig. 4).

- Constat identique pour les formations holocènes de Pont de Joux dont la base de la coupe datée à $7180 \pm$ 150 BP montre un dispositif latéralement transgressif sur la bordure du talweg (fig. 5). 


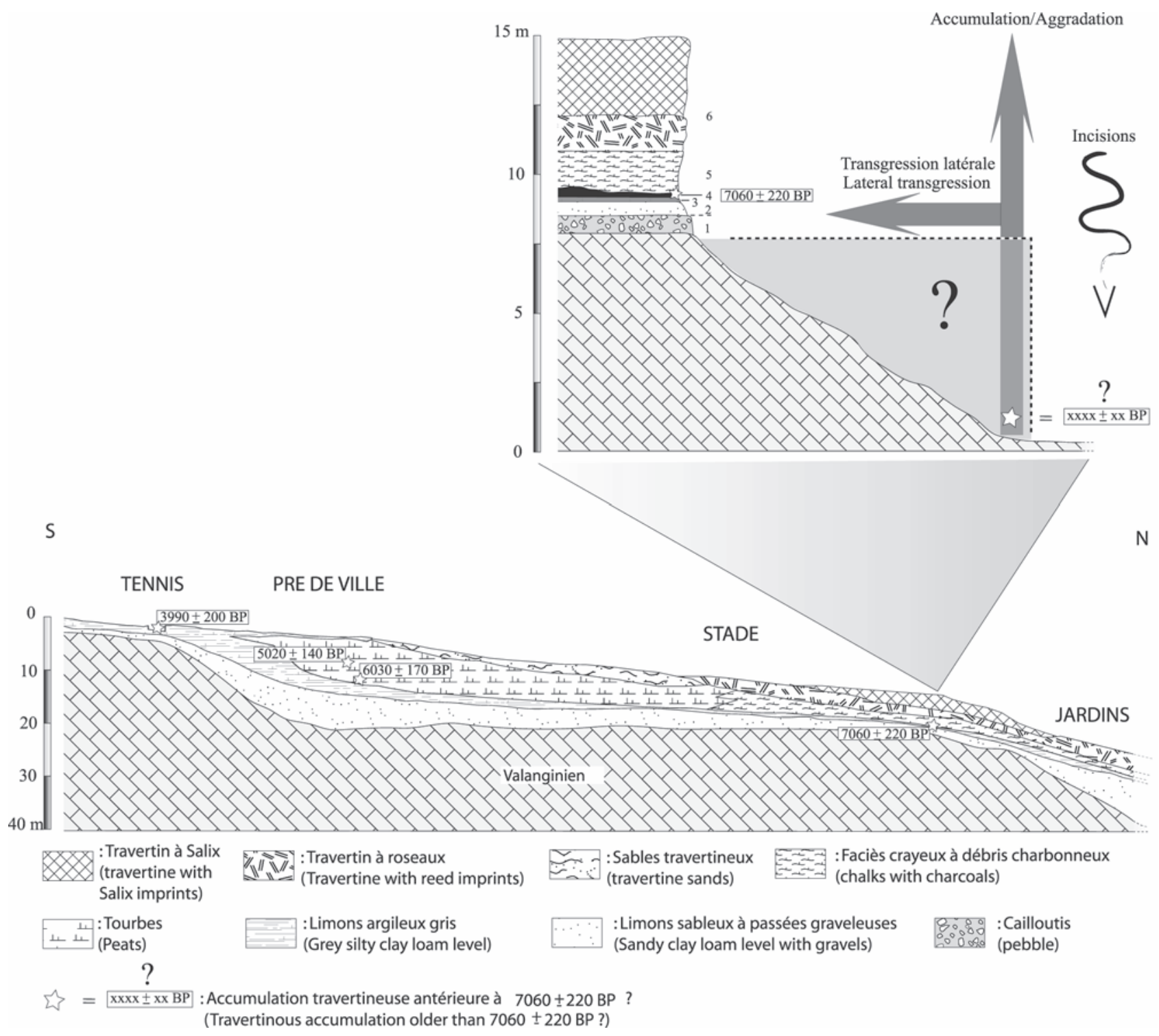

Fig. 4 : La séquence travertineuse holocène de Meyrargues (Bouches du Rhône), position géomorphologique et détail sur la base de la formation au niveau du canal EDF.

Fig. 4: The Holocene travertine formation of Meyrargues, Bouches du Rhône, geomorphological position and detailed view of the basis near the "canal EDF".

- Le cas du Serre de Montdenier, illustre particulièrement notre propos. Dans la coupe aval du bassin, la succession des unités stratigraphiques démontre également le caractère latéralement transgressif de ce type de formation avec un niveau travertineux à empreintes de mousses daté à $7760 \pm 80$ BP reposant directement sur les formations de versants à gélifracts (Nevière, 1996). Dans la coupe amont, située à proximité de l'axe du chenal, plusieurs datations récemment réalisées (Roiron et al., sous presse) comprises entre $9780 \pm 200 \mathrm{BP}(\mathrm{U} / \mathrm{Th})$ et $8480 \pm 50 \mathrm{BP}$ $\left({ }^{14} \mathrm{C}\right)$ indiquent finalement un démarrage de l'accumulation carbonatée remontant au moins au Préboréal.

- Enfin on peut citer la partie supérieure de la séquence holocène du Mirail qui se caractérise par plusieurs incisions mineures intraformationelles (cf. infra et fig. 3). Chaque fois, les nouveaux dépôts qui cicatrisent ces petits talwegs creusés dans les formations antérieures reproduisent le dispositif latéralement transgressif. C'est le cas de la coupe du Verger-nord illustrée par la figure $n^{\circ} 6$. C'est également le cas de la coupe de la Sarrière où une accumulation travertineuse construite entre $1880 \pm 30 \mathrm{BP}$ et $1775 \pm 30 \mathrm{BP}$, transgresse sur les niveaux crayeux et détritiques latéraux dont la base est datée à $7400 \pm 50 \mathrm{BP}$ (fig. 3).

Ces deux exemples montrent le caractère répétitif dans le temps et à toutes les échelles de cette organisation des différents faciès travertineux en fonction des variations paléohydrologiques et de leurs impacts morphodynamiques.

L'environnement morphosédimentaire joue aussi un rôle important dans la conservation des données 


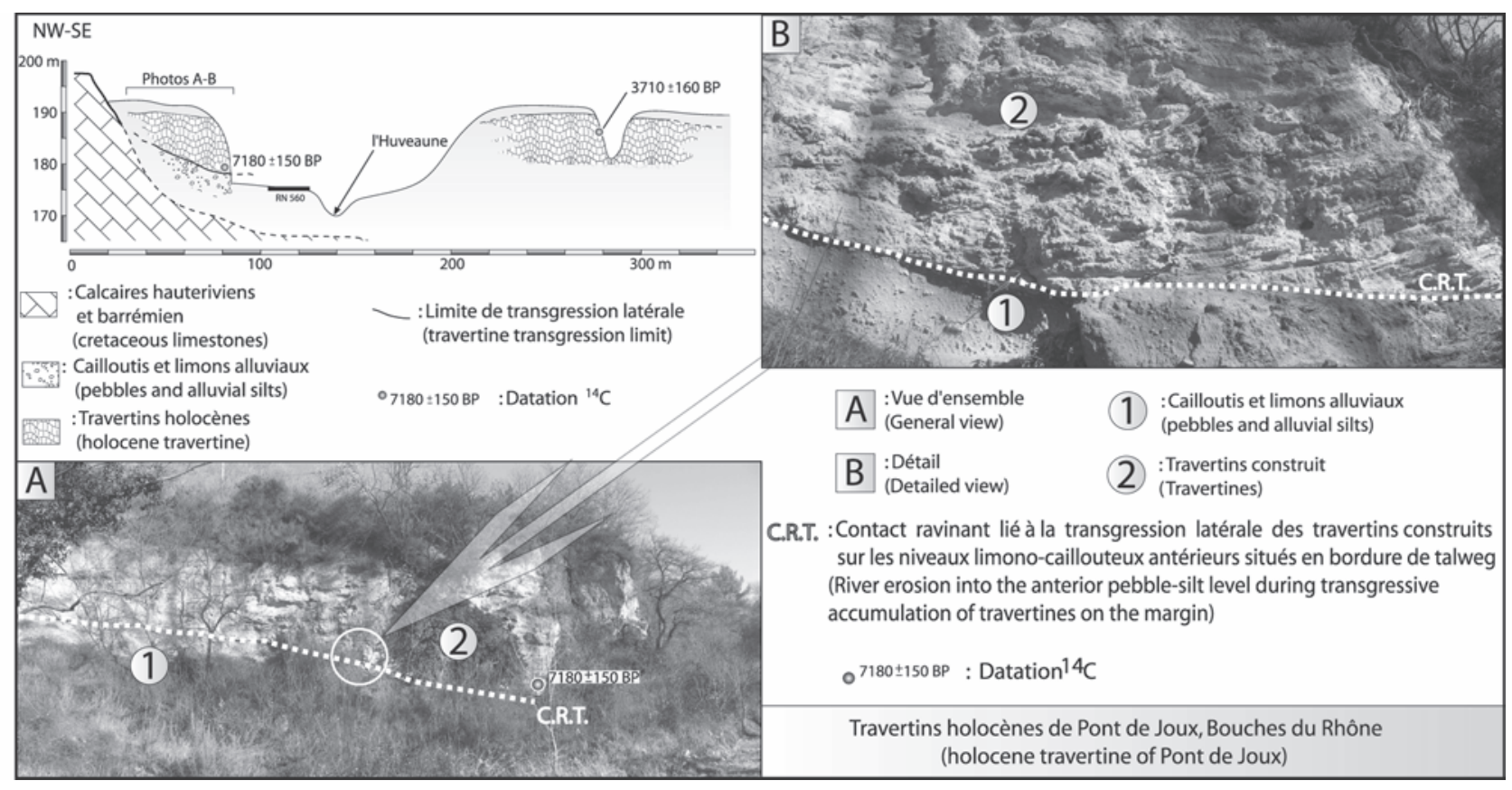

Fig. 5 : La formation travertineuse holocène de Pont de Joux (Bouches du Rhône), transgression des faciès construit sur les marges du talweg. Fig. 5: The Holocene travertine formation of Pont de Joux, Bouches du Rhône, transgressive situation on the talweg margin.

stratigraphiques. En domaine montagnard d'altitude par exemple, la conjugaison d'une végétation particulièrement ouverte (dominée par les pelouses), de fortes pentes et de conditions climatiques drastiques favorise l'instabilité des versants et notamment la production de glissements de terrain. Les séquences travertineuses y sont lacunaires et discontinues, particulièrement exposées aux agents de l'érosion, à l'image des formations de l'Aigue Agnelle dans le Queyras (Ali et al., 2002). A la sensibilité interne des systèmes travertineux s'ajoute donc ici celle du milieu montagnard. Ces paramètres en interaction sont entraînés dans une dynamique couplée enregistrant de façon remarquable les perturbations environnementales. Dans certains cas, les lacunes observées peuvent être des indicateurs particulièrement pertinents de l'évolution de ce type de milieux ou de variations plus globales d'origine exogène.

Ces multiples exemples reflètent la complexité des relations entre les différents facteurs impliqués dans le développement des systèmes travertineux du Midi méditerranéen. Lors des synthèses antérieures ces systèmes étaient définis comme diachroniques (formations qui semblaient débuter dans certains secteurs au Préboréal et dans d'autres à l'Atlantique) et discontinus (séquences incomplètes débutant au Préboréal et s'achevant postérieurement au Néolithique Final) alors qu'en définitive, globalement, le démarrage de l'accumulation travertineuse semble s'amorcer dès le Tardiglaciaire et s'achever au Petit Age Glaciaire. Certaines discontinuités et diachronismes relèveraient alors du contexte morphologique et hydrologique ou de la sensibilité du milieu.

\section{4 - INTERPRÉTATIONS DES FACIÈS ET DES DYNAMIQUES MORPHOSÉDIMENTAIRES}

\section{1 - LES DIFFÉRENTS FACIÈS ET LES MILIEUX QUI LEURS SONT ASSOCIÉS}

Les différents contextes environnementaux dans lesquels se développent les formations travertineuses, de même que l'impact des fluctuations morphosédimentaires et paléohydrologiques, génèrent une variété de faciès significative des conditions de dépôts.

Plusieurs types de faciès sédimentaires peuvent être distingués :

- Les travertins construits, qui résultent de l'accumulation de tapis algo-bactériens calcigènes. Bien indurés, avec des faciès stromatolithiques rubanés ou laminaires lorsqu'ils sont les plus purs (travertins stricto sensu, Vaudour, 1986b), ils peuvent être plus tendres, poreux ou vacuolaires. Ils sont assimilés dans ces derniers cas à des tufs calcaires (Vaudour, 1986b). Fossilisant fréquemment de nombreux végétaux (feuilles, aiguilles, cônes, troncs, mousses, brindilles, etc...) leur morphologie (bancs, banquettes, vasques, ressauts, cascades, dômes, etc...) est fortement dépendante du support sur lequel ils se développent. Ces faciès sont associés à des milieux de fort hydrodynamisme (ruisseaux, torrents, chutes d'eau, ruissellements sur versants). Ils se localisent préférentiellement au niveau des ruptures de pente ou d'embâcles (rochers, branches, etc...) qui accentuent, par leur brassage, le dégazage des eaux et accélèrent la sursaturation des solutions responsables de la précipitation des carbonates (Lu, 2000). Les 
édifices construits peuvent aboutir dans certains cas à la formation de barrages à l'amont desquels se développent des zones palustres ou des étendues lacustres.

- Une place à part doit être réservée aux faciès gélifractés, spécifiques des formations d'altitude. Ce sont à l'origine des travertins construits laminés, micro-fracturés au fur et à mesure de leur élaboration par le jeu de l'alternance gel/dégel (Fort, 1981 ; Mlakar et al., 1999). Les concrétionnements fragmentés peuvent subir un transport (gravitaire ou lié au ruissellement) sur une courte distance, et font l'objet d'une cimentation calcitique synsédimentaire dont résultent les faciès micro-bréchiques. L'analyse comparative de formations actuelles en milieu montagnard indique qu'ils se développent préférentiellement dans des zones de faible ruissellement dépourvues de couverture forestière protectrice (qui amoindrie les effets de l'onde de gel), ce qui qualifie ces faciès comme caractéristiques des milieux ouverts d'altitude.

- Les craies calcaires, qui sont des faciès meubles généralement blanchâtres ou grisâtres liés à la précipitation de fines particules carbonatées (Geurts, 1976), sont parfois litées en alternance avec de fins niveaux charbonneux, des incrustations oncolithiques (autour d'un noyau concentrique), en tubes (encroûtement de débris végétaux), choux-fleurs ou plaques en fonction de l'énergie des écoulements et du type de milieu concerné (alluvial, palustre ou lacustre). Ces dépôts sont symptomatiques de contextes environnementaux de faible hydrodynamisme, localisés dans des vallées larges et étalées, en amont de barrages travertineux ou latéralement au chenal principal d'écoulement dans le cas des systèmes carbonatés fluviatiles.

Aux faciès travertineux sensu stricto, qui représentent des milieux relativement stables favorables à leur accumulation, sont associés des faciès mixtes ou intégralement terrigènes qui appartiennent toutefois à la séquence travertineuse type. Il est possible de distinguer :

- Les ensembles travertino-détritiques qui sont constitués soit d'éléments détritiques allochtones (argiles, limons, cailloutis) mêlés à des concrétionnements autochtones (laminés, à mousses, crayeux, oncolithiques), soit d'éléments clastiques essentiellement travertineux (sables travertineux). Ces derniers proviennent de l'érosion et du remaniement de formations carbonatées en développement. Tous ces dépôts dénotent de milieux fragilisés ou particulièrement sensibles.

- Les faciès purement détritiques, constitués de sédiments argileux, limoneux, sableux ou caillouteux (éléments du substrat parfois mêlés, en proportion largement inférieure aux ensembles travertino-détritiques, à des blocs ou fragments de travertins) traduisent des érosions importantes sur les versants et révèlent des épisodes de crises morpho-sédimentaires, de déséquilibres, d'instabilités.

\section{2 - EVOLUTION CYCLIQUE DE LA SÉQUENCE CARBONATÉE : UNE SUCCESSION DE RELATIONS ENTRE TRAVERTINS, DÉTRITISME ET INCISIONS}

Une typologie cyclique de l'évolution verticale de la séquence travertineuse a déjà été élaborée (Vaudour, 1986b ; Magnin et al., 1991). Celle-ci définissait deux principaux pôles d'évolution, l'un positif concernant la partie inférieure des formations l'autre négatif dans la deuxième moitié de la coupe stratigraphique, annonçant la «mort » progressive de l'édifice travertineux (Magnin et al., 1991). Dans le premier pôle, l'enchâ̂nement des faciès débutait par des cailloutis alluviaux (parfois des blocs éboulés et glissés comme à St Antonin, Guendon \& Vaudour, 1981 et fig. 7), et se poursuivait par des limons ou des argiles, des craies travertineuses puis des travertins construits (principal corps carbonaté de la formation). Le second pôle s'amorçait par des concrétionnements remaniés (sables travertineux, limons à manchons calcitiques, etc...) et était souvent recouvert par une nappe détritique colluviale. Une incision plus ou moins importante suivait généralement la mise en place de ces dépôts, avant que ne se réalise un nouveau cycle sédimentaire travertineux, emboîté ou étagé par rapport au précédent (Magnin et al., 1991). Cette analyse traduisait une évolution environnementale et morphosédimentaire qui commençait par des conditions de milieux essentiellement détritiques où la travertinisation n'avait pas la possibilité de s'exprimer et de s'accumuler. L'apparition d'un contexte carbonaté au préalable crayeux, qui constituait les premiers stades de l'accumulation travertineuse, suivit de faciès construits (travertins laminés ou rubanés, incrustations recouvrant divers débris végétaux, etc...) représentait au final l'optimum de la carbonatogenèse.

Sans pour autant remettre totalement en cause ce schéma de référence où la succession des faciès définie se vérifie régulièrement sur le terrain, les derniers travaux réalisés à St Antonin (Guendon et al., 2003), dans le Queyras (Ali et al., 2003) et dans le Luberon (Ollivier et al., 2004, 2006) semblent apporter de nouvelles précisions. Celles-ci portent notamment sur l'opposition qui semblerait exister entre détritisme et travertinisation ainsi que sur la signification séquentielle des faciès travertineux crayeux et construits.

A St Antonin sur le versant sud de la St Victoire (Guendon et al., 2003) ou dans le Mirail, sur le piémont méridional du Grand Luberon (Ollivier et al., 2004) pour utiliser deux cas complémentaires (l'un de type cascadant au fort hydrodynamisme, l'autre caractéristique de milieu palustre moins dynamique), le détritisme ne semble pas gêner l'accumulation travertineuse. Le cas de St Antonin est particulièrement représentatif. Le caractère synchrone et la mixité entre éléments détritiques et carbonatation au cours des premières phases de dépôt datées du Préboréal ne fait aucun doute. Seule l'intervention d'une péjoration climatique au Boréal additionnée à la sensibilité propre des systèmes travertineux semble pouvoir interrompre 
momentanément l'accumulation des travertins. En dernière partie de séquence, dans un contexte où les occupations humaines de la fin du Néolithique sont de plus en plus prononcées, épisodes détritiques et phases carbonatées coexistent également. Les différents faciès se déposent en alternance ou parfois dans le même intervalle de temps. Dans ce dernier cas se constitue un pôle sédimentaire mixte composé de lentilles travertineuses construites ou à oncolithes, de limons, de sables et de cailloutis. Au Mirail, dans le Luberon, le détritisme est là aussi souvent mêlé à l'accumulation des travertins que ce soit dans les craies à la faveur d'épisodes de crues (parfois, plutôt de migrations latérales du chenal principal) ou préalablement aux faciès construits à la suite d'une phase d'incision plus ou moins importante. Dans le premier, cas il s'agira de la simple expression du fonctionnement autocyclique du système, dans le second, de la manifestation de brèves perturbations d'origine exogène.

Toujours dans le Luberon, la principale accumulation travertineuse postglaciaire, du Tardiglaciaire à la seconde moitié de l'Atlantique, est exclusivement représentée par des faciès crayeux entrecoupés de lentilles détritiques et de sols hydromorphes. A partir du Néolithique Final les premières incisions entaillent brutalement les niveaux crayeux sous jacents et rompent la continuité de la dynamique plurimillénaire d'accumulation carbonatée (fig. 3). De telles ruptures, signe d'une certaine instabilité du milieu, se reproduisent fréquemment de la fin du Néolithique à l'époque historique sans pour autant interrompre de façon définitive le développement des formations, phénomène qui se produira, nous l'avons vu, au Petit Age Glaciaire. Immédiatement à la suite de l'incision dans les craies, un matériel détritique relativement grossier se met en place. Celui-ci peut se composer de blocs volumineux (jusqu'à $50 \mathrm{~cm}$ de grand axe), de cailloutis hétérométriques sub-émoussés, et bien souvent de fragments de céramique et d'ossements d'animaux remaniés, témoignages des occupations humaines locales du Néolithique Final. Ce matériel, souvent déjà encroûté lors de son dépôt, laisse systématiquement la place à des faciès construits stromatolithiques qui transgressent latéralement sur les berges ou les zones palustres proximales (fig. 6). L'apparition de ces faciès construits est due aux nouvelles conditions générées par l'incision qui accentue la pente et la concentration des écoulements, augmente l'hydrodynamisme, le brassage des eaux et le dégazage du $\mathrm{CO}_{2}$. Une fois le chenal comblé et la pente régularisée, le contexte hydrodynamique redevient plus calme et l'on assiste à la réapparition de l'accumulation crayeuse. Ces faciès à craies travertineuses sont donc significatifs d'un retour à des conditions environnementales plus stables. Cette notion de stabilité, d'équilibre des milieux, est un élément clé dans la phase de développement optimale

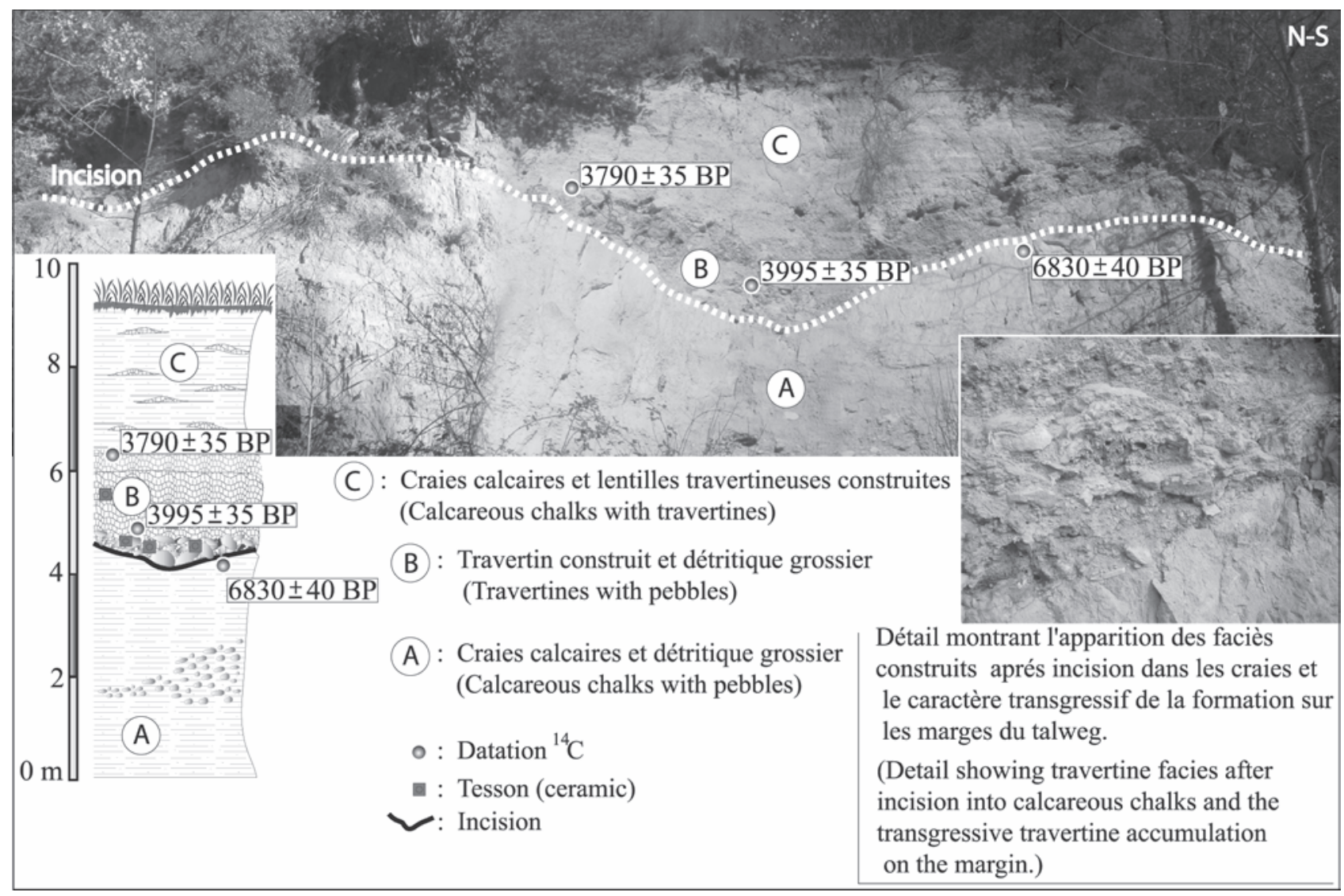

Fig. 6 : Coupe du Verger-nord (formation travertineuse du Mirail, Luberon, Vaucluse), variations morphosédimentaires et transgression sur les marges.

Fig. 6: Verger-nord sequence in the Postglacial travertine formation of the Luberon, Vaucluse, morphosedimentary variations and transgressive situation on the margin. 


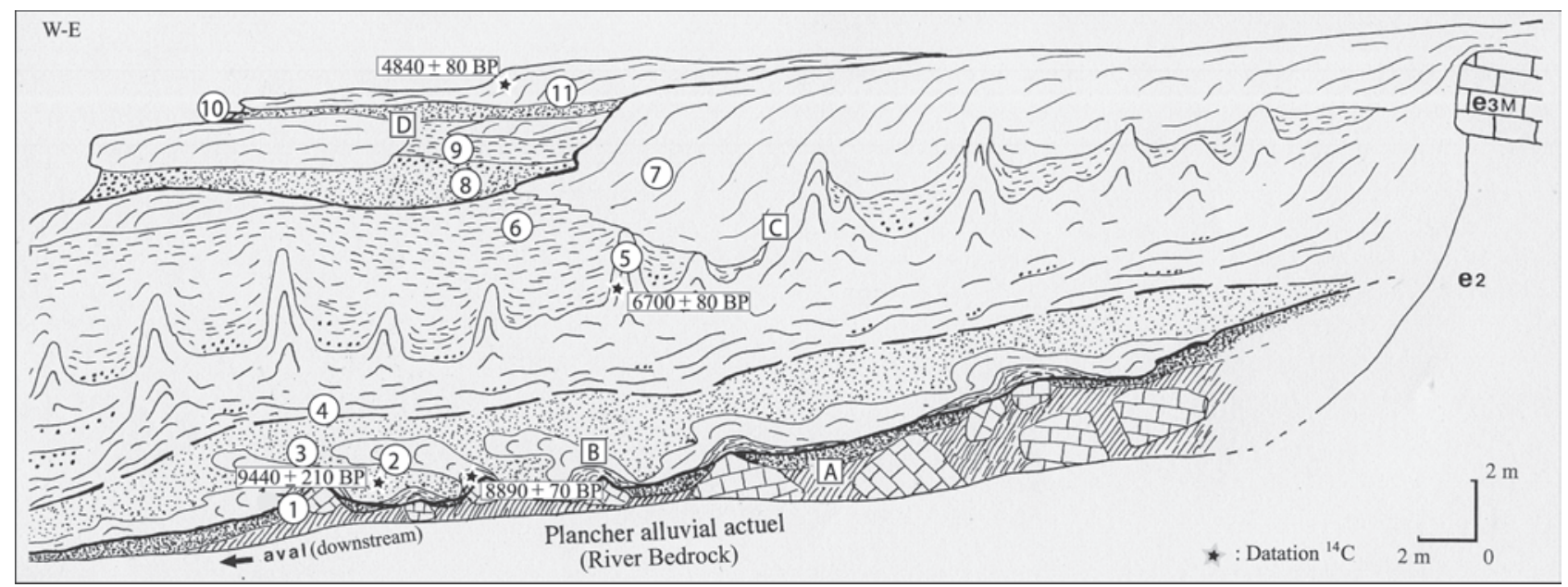

Fig. 7 : Coupe d'ensemble des travertins holocènes de la cascade de St Antonin (Bouches du Rhône) d'après Guendon et al., 2003.

Substratum : e2) Argilites rouges rouges du Paléocène moyen (Thanétien); e3M) Calcaire du Paléocène supérieur (Sparnacien).

Ensemble A : Blocs calcaires et dépôts alluviaux.

Ensemble $\mathrm{B}:$ 1) Travertins laminés ; 2) Travertins à mousses, 3) Limon rouge.

Ensemble C : 4) Travertin feuilleté ; 5) Gours à travertin laminé ; 6) Craies ; 7) Cascade de travertin.

Ensemble D : 8) Niveau détritique contenant quelques vestiges remaniés du Néolithique ; 9) Travertin caverneux et limons crayeux ; 10) Niveau détritique ; 11) Travertin vacuolaire.

Fig. 7: Schematic representation of the St Antonin Holocene travertine according to Guendon et al., 2003.

Substratum : e2) Palaeocene marl (Thanatian); e3M) Palaeocene limestone (Sparnacian).

Unit A : Block limestone and alluvial deposits.

Unit B : 1) Laminated travertine ; 2) Moss travertine ; 3) Red silt.

Unit C : 4) Laminated travertine ; 5) Rimstone bar system with laminated travertine ; 6) Chalk ; 7) Old travertine fall.

Unit D : 8) Brown clays and gravels with archaeological remains ; 9) Friable travertine and chalk ; 10) Brown clays ; 11) Friable travertine.

de la travertinisation. Les futures ruptures entraîneront par la suite la répétition de ce même cycle sédimentaire carbonaté de référence, défini par l'enchaînement caractéristique des dynamiques et des faciès en quatre étapes selon le mode: incision-détritique-travertin construit-retour aux conditions initiales (dans notre cas il s'agira des craies travertineuses).

Même s'il existe une multitude de situations intermédiaires, deux principaux types d'évolutions verticales sont donc à distinguer en fonction de l'environnement morphosédimentaire :

- Dans les formations de cascade, de barrage, à fort hydrodynamisme lié à la morphologie des sites ( $\mathrm{St}$ Antonin, fig. 7, Vauvenargues, St Guilhem du Désert par exemple), les faciès construits et stromatolithiques prédominent. Ils coexistent souvent avec des épisodes crayeux et des décharges détritiques. Dans ce cas, les niveaux mixtes composés d'éléments caillouteux et sableux mêlés à des incrustations carbonatées constitueraient, en base de séquence une phase de transition vers des faciès travertineux plus purs représentant un optimum de stabilité morphosédimentaire, en sommet de coupe un premier stade de dégradation de l'environnement.

- Dans les zones palustres alluviales à faible hydrodynamisme les faciès crayeux s'expriment majoritairement. Les travertins construits n'apparaissent alors que ponctuellement à la faveur d'incisions qui dynamisent les écoulements (fig. 6). Dans ce cas les faciès construits accompagneraient et seraient consécutifs de périodes d'instabilité morphosédimentaires.

\section{3 - LES TRAVERTINS : DES FORMATIONS SENSIBLES EN INTIME RELATION AVEC LES MODIFICATIONS DU BIOTOPE}

Des analyses paléoécologiques (anthracologie et malacologie) ont été associées aux études sédimentologiques et géomorphologiques. Elles concernent les formations de St Antonin et du Mirail à l'évolution globale particulièrement explicite. Les résultats obtenus par ces recherches témoignent de la nécessité d'une conjonction des facteurs pour engendrer la «mort» progressive des édifices travertineux.

A St Antonin (Guendon et al., 2003), la séquence débute au Préboréal-Boréal dans un contexte forestier ouvert (espèces hygrophiles et pionnières de ripisylve et quelques essences mésophiles de la chênaie pubescente) où la travertinisation construite se développe conjointement à un détritisme de départ en diminution progressive. Ensuite, de la fin du Boréal au début de l'Atlantique, le milieu végétal se referme (chênaie pubescente), l'accumulation des travertins devient optimale (notamment selon la relation : densification du couvert végétal, augmentation du $\mathrm{CO}_{2}$ favorable à la dissolution du substratum calcaire, transport en solution et re-précipitation des carbonates sous la forme de travertins). Celle ci perdure malgré une première ouverture du milieu végétal dès l'Atlantique ancien. Enfin, du milieu de l'Atlantique jusqu' au Subboréal, le contexte environnemental passe d'un milieu forestier ouvert (chênaie pubescente et ronce) à un milieu forestier très ouvert (chênaie pubescente, Pin d'Alep, Genévrier et ronce). L'apparition brutale d'érosions et le retour d'un important détritisme, associé à un mobilier 


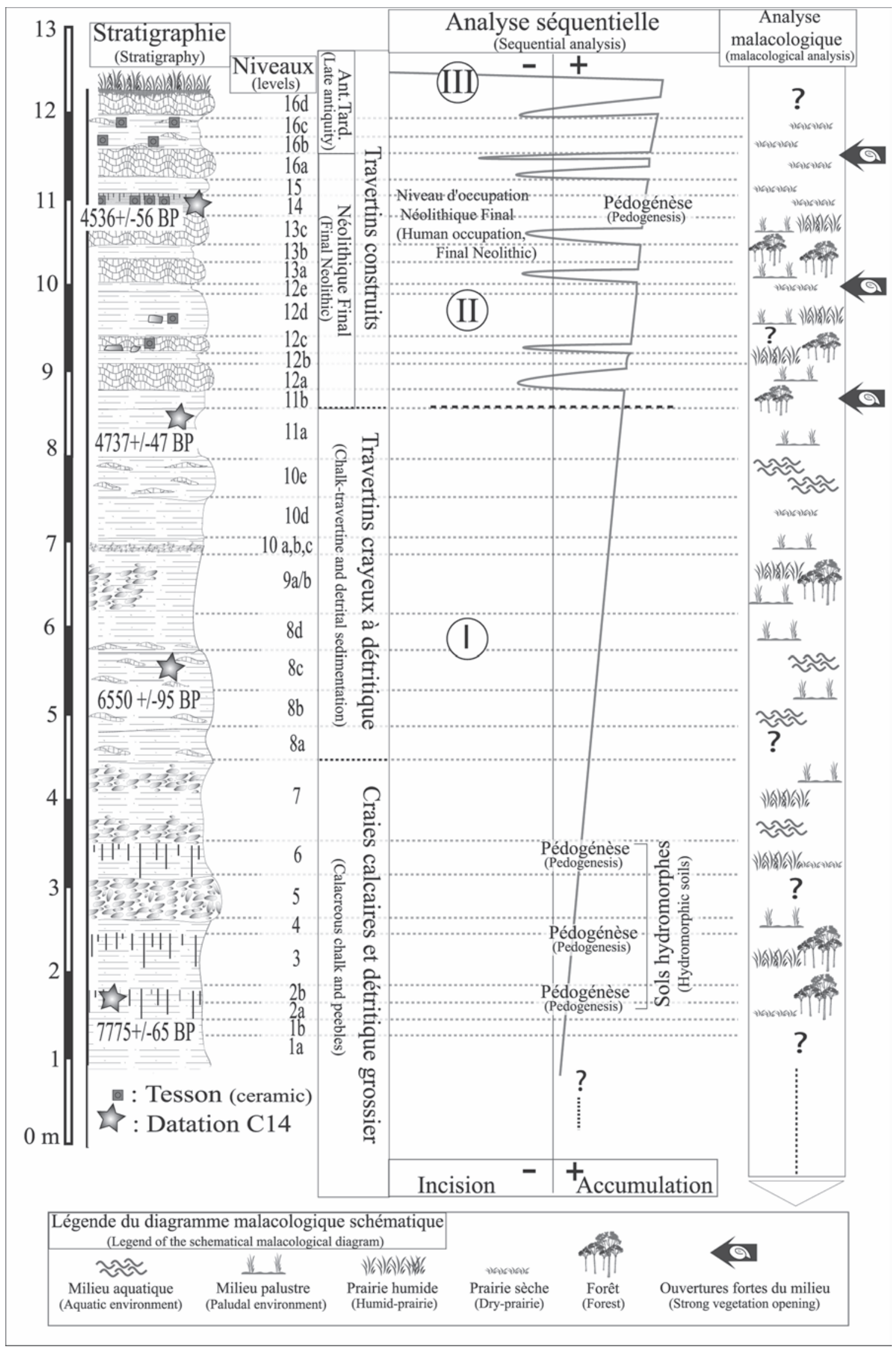

Fig. 8 : Analyse séquentielle et paléoécologique de la coupe du Verger-sud (formation travertineuse postglaciaire du Mirail, Luberon, Vaucluse). I) Accumulation continue, contexte environnemental stable ; II) Accumulation discontinue, ruptures, incisions, changements de faciès, instabilité environnementale ; III) Cumul des pressions climato-anthropiques, incision linéaire généralisée des talwegs.

Fig. 8: Sequential and palaeoecological analysis of the Verger-sud sequence, Luberon, Vaucluse.

I) Continuous accumulation, environmental stability ; II) Discontinuous accumulation, incision, facies changes, environmental instability ; III) Cumulated effect of climatic and anthropogenic pressure, generalised talweg linear incision. 


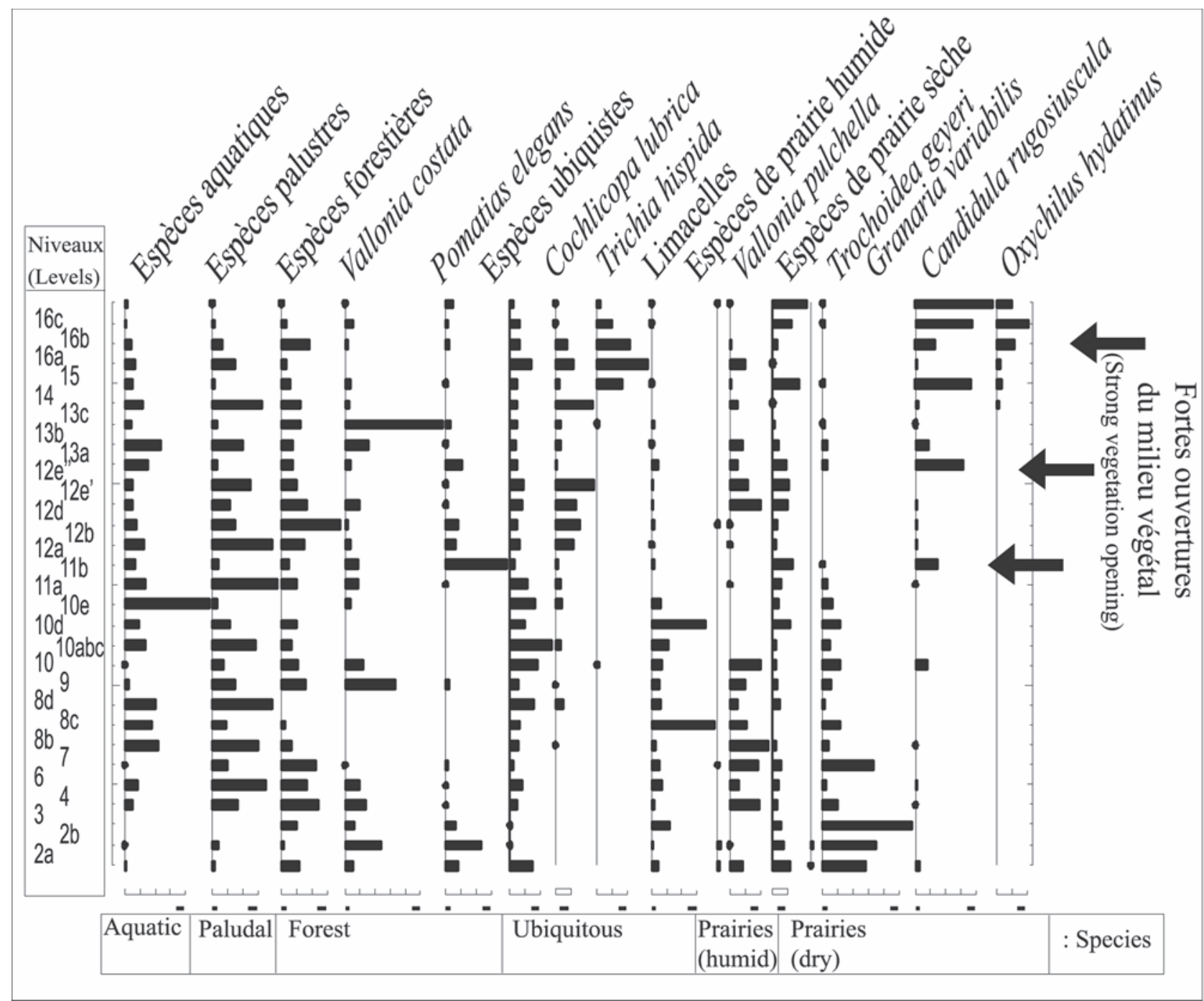

Fig. 9 : Diagramme malacologique de coupe du Verger-sud (formation travertineuse postglaciaire du Mirail, Luberon, Vaucluse), d'après les analyses de Martin, 2004.

Fig. 9: Malacological diagram of the Verger-sud sequence (Postglacial travertinous Mirail formation, Luberon, Vaucluse) according to Martin, 2004.

archéologique remanié datant du Néolithique Final, semble s'accorder avec l'ouverture croissante du milieu végétal observé par les analyses anthracologiques et malacologiques. Toutefois, en sommet de séquence, la travertinisation construite reprend.

Dans le Luberon (Ollivier et al., 2006), la formation du Mirail connaît une évolution en de nombreux points comparable à celle de St Antonin. L'accumulation travertineuse y débute au Tardiglaciaire, mais les études malacologiques concernant cette période sont en cours de réalisation par Sophie Martin et Frédéric Magnin (I.M.E.P., Aix en Provence). A l'Atlantique, les faciès crayeux se développent dans un environnement immédiat palustre bordé d'une forêt ouverte (Martin, 2004, fig. 8 et fig. 9). L'existence de sols hydromorphes suggère des conditions édaphiques assez stables (fig. 8). Seules quelques nappes caillouteuses provenant de petites crues ou de migrations latérales du chenal principal rappellent le caractère alluvial du fond de vallon. Ces décharges détritiques ne sont pas accompagnées d'incisions et n'entravent pas l'accumulation des craies. L'alternance entre milieux typiquement palustres et reconquêtes forestières se poursuit jusqu'à la fin de l'Atlantique sans modification notable des faciès travertineux. Au Subboréal, de fortes ouvertures du milieu végétal interviennent (fig. 8 et fig. 9). Dans le même temps, les premiers faciès travertineux construits apparaissent à la suite de petites incisions dans les craies. Conjointement on observe une installation marquée des populations du Néolithique Final dans le secteur, manifestée par de nombreux vestiges archéologiques et un niveau d'occupation humaine. En sommet de séquence, la travertinisation construite se poursuit au moins jusqu'au Moyen Age chaque fois associée à de petites phases d'incisions.

Du Tardiglaciaire à la seconde moitié de l'Atlantique, à St Antonin comme dans le Luberon, les modifications dans l'accumulation carbonatée semblent inféodées aux seules variations des conditions bioclimatiques. Cependant, les fluctuations climatiques ne sont pas suffisamment prononcées pour perturber durablement et interrompre le développement des séquences carbonatées. En revanche, dès la fin de 1'Atlantique/début du Subboréal, les variations bioclimatiques (Magny et al., 2006) associées à l'impact de plus en plus répétitif des occupations humaines se font 
ressentir. Elles se manifestent par des ouvertures du milieu végétal nettement marquées et par des ruptures dans les séquences travertineuses: des incisions, notamment dans le Luberon, et des changements de faciès dans les deux sites (détritisme à St Antonin, travertin construit dans le Luberon).

\section{5 - CONCLUSION}

Les études récentes ont permis quelques révisions ou ajouts concernant certains concepts établis à partir de l'étude des nombreuses formations travertineuses emblématiques du sud de la France.

- L'accumulation carbonatée démarre dès le Tardiglaciaire et non plus uniquement au Préboréal ou à l'Atlantique. Elle s'achève postérieurement au Moyen Age. Le démantèlement des édifices travertineux et l'instauration de la dynamique d'incision linéaire des talwegs (qui concerne également tous les types de formations alluviales) se déroulent au Petit Age Glaciaire entre le XIII ${ }^{\text {ème }}$ et le XVII ${ }^{\text {ème }}$ siècle après Jésus Christ. L'origine exacte de cette rupture majeure reste toutefois à déterminer.

- L'interprétation des formations comme débutant à l'Atlantique ou de façon diachronique selon les régions résulterait du contexte géomorphologique, du type de milieu, et de la position de la coupe stratigraphique étudiée par rapport au talweg. En milieu stable (Luberon) ou en fonction des conditions d'affleurement, on observe des séquences complètes du Tardiglaciaire au Moyen Age (malgré quelques incisions intraformationnelles). En milieu sensible (montagnard) ou selon de mauvaises conditions d'affleurement (vallées étroites où seules les coupes de bordure de talweg subsistent), les séquences sont fortement discontinues donnant l'image d'un démarrage de la travertinisation plus tardif ou diachronique. Ce diagnostic est renforcé par le caractère transgressif des phases construites sur les marges qui, en recouvrant celles-ci après avoir rapidement comblé le chenal principal, rajeunissent les formations latérales.

- Les faciès travertineux, qu'ils soient crayeux ou construits peuvent coexister avec le détritisme sans inhibition de la carbonatation. Dans les ensembles palustres-alluviaux, les craies peuvent être considérées comme représentatives d'une période optimale de stabilité du milieu. Souvent, les faciès construits, synonymes d'augmentation de l'hydrodynamisme, apparaissent à la suite de petites incisions dans les niveaux crayeux sous jacents. Dans ce cas précis, la travertinisation construite intervient après une phase d'instabilité morphogénique, de rupture dans le processus d'accumulation. Elle ne signe donc pas forcément un optimum de la carbonatation mais plutôt une étape intermédiaire vers un nouvel équilibre en faveur de la croissance des édifices.
- Enfin, du Tardiglaciaire à la seconde moitié de l'Atlantique, le développement des formations semble sous l'influence majeure des conditions bioclimatiques et l'on observe une bonne corrélation entre les dynamiques végétales et la travertinisation. Dès le Néolithique Final, dans un contexte d'augmentation des occupations humaines, les séquences enregistrent de nombreuses ruptures qui ne modifient pas la tendance générale à l'accumulation des travertins. Ces perturbations sont accompagnées de changements de faciès et d'ouvertures fortes du milieu végétal.

La séquence travertineuse apparaît donc comme un système complexe, sorte d'autopoïèse (capacité d'un système à se produire et à s'auto-entretenir, Varela, 1989), où chaque perturbation interne ou externe est suivie d'une régénération de l'ensemble selon de multiples formes et en fonction de l'état initial (défini par ses caractéristiques géomorphologiques). Le moindre relâchement d'une des pressions (climatique ou anthropique) sur ces environnements sensibles peut favoriser le retour de l'accumulation carbonatée et la croissance des édifices. Seul le poids plurimillénaire des occupations humaines additionné aux changements climatiques récurrents de la seconde moitié de l'Holocène sont suffisants pour interrompre l'accumulation des travertins et démanteler les formations, phénomène probablement temporaire et tributaire du prochain renversement de tendance.

\section{REMERCIEMENTS}

Nous tenons particulièrement à remercier M André Muller, Directeur du programme de recherche « 10000 ans d'histoire sur le piémont méridional du Grand Luberon » dans lequel s'inscrit une bonne partie des nouveaux résultats obtenus. Nous remercions également le Parc naturel régional du Luberon, le Conseil Général du Vaucluse, le Service Régional de l'Archéologie P.A.C.A., et le Ministère de la Culture. Egalement merci à Mlle Jessica Hackett pour la relecture du résumé en anglais, ainsi qu'aux deux rapporteurs de cet article, Messieurs Michel Dubar et Jean Sommé, pour leur rapidité et la pertinence de leurs observations.

\section{RÉFÉRENCES BIBLIOGRAPHIQUES}

ALI A.-A., GUENDON J.-L., TERRAL J.-F., QUINIF Y., \& ROIRON P., 2002 - Végétation holocène et dynamique d'une forêt subalpine (Queyras, France) : étude géomorphologique et paléobotanique de formations travertineuses, Quaternaire, 13 (3-4), 229-236.

ALI A.-A., GUENDON J.-L., TERRAL J.-F., \& ROIRON P, 2003 - Les systèmes travertineux holocènes et les paléopaysages méditerranéens et subalpins (France) : une analyse géobotanique séquentielle à haute résolution spatiale, Géographie physique et Quaternaire, 57 (2-3), 219-235.

ALI A.-A., ROIRON P., GUENDON J.-L., \& TERRAL J.-F., 2004 - Subalpine vegetation dynamics in the southern French Alps during the Holocene : evidence from plant imprints and charcoal preserved in travertine sequences, Artic, Antartic and Alpine Research, 36 (1), 42-48.

AMBERT P., 1979-1981 - L'abri Rothschild (Cabrières-Hérault), Rapport de fouille de sauvetage, D.R.A.C., Languedoc-Roussillon, 58 p., (inédit). 
AMBERT P., 1982 - Recherches sur l'âge des tufs calcaires en Languedoc central, Phénomènes karstique III, Mémoires et documents du C.N.R.S., 171-179.

AMBERT P., 1988 - Les tufs de la Resclauze (Gabian-Hérault), leur évolution à l'Holocène, Les édifices travertineux et l'histoire de l'environnement dans le Midi de la France, U.A. 903 CNRS, Travaux 13, Aix en Provence, 99-106.

AMBERT P., 1991 - L'évolution géomorphologique du Languedoc central depuis le Néogène, Thèse d'Etat, Aix-Marseille II, tome 1, 224 p., tome 2, illustrations et cartes H.T.

AMBERT P. (dir.), 1993-1998 - Travertins et dépressions fermées de piémont : paléoenvironnements et anthropisation des paysages du midi méditerranéen, Rapport de synthèse G.D.R. 1058, 60 p., (inédit).

AMBERT P., 1997 - Travertins et dépressions fermées de piémont paléoenvironnements et anthropisation des paysages du midi méditerranéen, Etudes de Géographie Physique, supplément au $n^{\circ}$ XXVI, 31-34.

AMBERT P., \& DELGIOVINE A., 1979 - Les tufs de la Resclauze à Gabian, Archéologie en Languedoc, 2, 17-18.

AMBERT P., \& GASCO J., 1989 - Les tufs de Saint-Guilhem-LeDesert, évolution holocène et pression anthropique sur le milieu karstique (Languedoc, France), Bulletin du Musée d'Anthropologie et de Préhistoire de Monaco, 32, 63-85.

BAKALOWICZ M., 1988 - La formation des travertins : aspects géochimiques, essai de synthèse et discussion, Les édifices travertineux et l'histoire de l'environnement dans le Midi de la France, U.A. 903 CNRS, Travaux 13, Aix en Provence, 261-268.

BAKER A., \& SIMMS M.J., 1998 - Active deposition of calcareous tufa in Wessex, UK, and its implications for the "late-Holocene tufa decline". The Holocene, 8 (3), 359-365.

BIGOT-CORMIER F., BRAUCHER R., BOURLÈS D., GUGLIELMI Y., DUBAR M., \& STÉPHAN J.F., 2005 - Chronological constraints on processes leading to large active landslides, Earth and Planetary Science Letters, 235, 141-150.

BJÖRK S., WALKER M.J.C., CWYNAR L.C., JOHNSEN S.J., KNUDSEN K.-L., LOWE J.J., WOHLFARTH B., \& INTIMATE MEMBERS, 1998 - An event stratigraphy for the Last Termination in the North Atlantic region based on the Greenland ice-core record : a proposal by the INTIMATE group. Journal of Quaternary Science, 13 (4), 283-292.

BROCHIER J.E., 1988 - Cinq millénaires de sédimentation dans le marais holocène de la Resclauze (Gabian, Hérault), étude géoarchéologique, Travaux 13, Les édifices travertineux et l'histoire de l'environnement dans le Midi de la France, U.A. 903 CNRS et A.T.P. PIREN, Aix en Provence, 117-137.

BROCHIER J.E., 2002 - Sédimentations néolithiques. Un lien avec l'état du couvert végétal ? In E. Badal, J. Barnabeu, \& B. Mart (eds.), El paisaje en el Neolitico méditerranéo. SAGVNTVM, Papeles del Laboratorio de Arquéologia de Valencia, extra 5, 115127.

BROCHIER J.E., \& LIVACHE M., 2003 - Les niveaux à crans de l'abri du Rouet (Carry-Le-Rouet, Bouches du Rhône) et les industries pléni-tardiglaciaires du bassin bas-rhodanien, Préhistoire du sud-ouest, supplément 6, Actes de la table ronde de Montauban (Tarn et Garonne), Les pointes à cran dans les industries lithiques du Paléolithique supérieur récent de l'oscillation de Lascaux à l'oscillation de Bölling, E. Ladier Editions, 47-66.

BROTTO M., 1986 - Karst de gypse et accumulations de tufs en Queyras, Méditerranée, 1-2, 118-125.

BRUNETON H., DEVILLERS B., JORDA C., OLLIVIER V., MIRAMONT C., BLANCHEMANCHE P., BERGER J.-F., \& PROVANSAL M., 2002 - Relations entre paléohydrologie et morphogenèse des petits et moyens bassins versants en basse Provence et Languedoc oriental, In JP. Bravard \& M. Magny (eds), Les fleuves ont une histoire : Paléoenvironnements des rivières et des lacs français depuis 15000 ans. Editions Errance, 259-267.

CHEYLAN M., \& D'ANNA A., 1979 - Note sur la station chalcolithique de la cascade, Vauvenargues (Bouches du Rhône), Bulletin Archéologique de Provence, 4, 3-14.

COLLECTIF, 1981 - Formations carbonatées externes tufs et travertins, Mémoire 3, Association française de karstologie, Aix en Provence-Nimes, $216 \mathrm{p}$
D'ANNA A., \& COURTIN J., 1986 - Travertins holocènes et sites préhistoriques : exemples dans le Var et les Bouches-du-Rhône, Méditerranée, 1-2, 31-39.

D'ANNA A., EVIN J., GUENDON J-L., JAUBERT J., MAGNIN F., OTTO T., ROIRON P., THINON M., VERNET J-L., \& VAUDOUR J., 1988 - Les édifices travertineux d'Auriol (Pont de Joux), et de Roquevaire (St Vincent). Révision stratigraphique. Contribution à l'histoire de l'environnement dans la vallée de l'Huveaune (Bouches-du-Rhône) depuis 50000 ans, Les édifices travertineux et l'histoire de l'environnement dans le Midi de la France, U.A. 903 CNRS, Travaux 13, Aix en Provence, 17-36.

DEL GIOVINE A., 1986 - Les travertins holocènes de la cascade de Vauvenargues (Bouches-du-Rhône), Méditerranée, 1-2, 81-91.

DIAZ DEL OLMO F., GUENDON J.L., BAENA ESCUDERO R., \& DELANNOY J.J., 1997 - Crono-sequencias de travertinos recientes : estudios comparado del S.E. de Francia y S. de Espana. Etudes de Géographie Physique, supplément XXVI, Aix en Provence, 95-97.

DUBAR M., \& CLAPPIER P., 1989 - Ségriès - Moustiers Sainte Marie (Alpes de Haute Provence) : un site préhistorique remarquable de l'avant-pays alpin. Bulletin Archéologique de Provence, 18, 1-6.

FORT M., 1981 - Les travertins de Samdo (Himalaya du Népal) : un exemple de concretionnement carbonaté en haute altitude. Actes du colloque de l'Association Géographique Française, "Formations carbonatées externes, tufs et travertins", Paris, 9 mai 1981, $79-88$

FREYTET P., \& FORT M., 1980 - Les formations plio-quaternaires de la Kali Gandaki et du bassin de Pokhara (Himalaya du Népal). Bulletin de l'Association Géographique Française, 471, 249-257.

GAUTIER E., 1992 - Recherches sur la morphologie et la dynamique fluviale dans le bassin du Buëch (Alpes du sud). Thèse de Géographie, Université Paris-Nanterre, 439 p.

GEURTS M.A., 1976 - Genèse et stratigraphie des travertins de fond de vallée en Belgique. Acta Géographica Lovaniensia, 16, 92 p.

GUENDON J-L., ALI A.A., ROIRON P., TERRAL JF., D'ANNA A., DIAZ DEL OMO F., \& BAENA ESCUDERO R., 2003 - Les travertins de St Antonin : Séquence géobotanique et climato-anthropique holocène. Karstologia, 41, 1-14

GUENDON J-L., \& VAUDOUR J., 1981 - Les "tufs" holocènes de St Antonin sur Bayon (Bouches-du-Rhône) : aspects petrographiques et significations paléogéographiques. Mémoire 3, Association française de karstologie, Aix en Provence-Nimes, 89-99.

INTIMATE MEMBERS - http : //www.geog.uu.nl/fg/INTIMATE/

JORDA M., MIRAMONT C., ROSIQUE T., \& SIVAN O., 2002 Evolution de l'hydrosystème durancien (Alpes du sud, France), depuis la fin du Pléniglaciaire supérieur. In JP. Bravard \& M. Magny (eds), Les fleuves ont une histoire: Paléoenvironnements des rivières et des lacs français depuis 15000 ans. Editions Errance, 239-249.

LANG J., \& LUCAS G., 1970 - Contribution à l'étude de biohermes continentaux: barrages des lacs de Band-e-Amir (Afghanistan central). Bulletin de la Société Géologique de France, 7 (XII-5), 834-842.

LOWE J.J., HOEK W.Z., \& INTIMATE GROUP, 2001 - Interregional correlation of palaeoclimatic records for the Last GlacialInterglacial Transition : a protocol for improved precision recommended by the INTIMATE project group. Quaternary Science Reviews, 20, 1175-1187.

LU G., ZHENG C., DONAHOE R.J., \& BERRY LYONS W., 2000 - Controlling processes in a $\mathrm{CaCO} 3$ precipitating stream in Huanglong Natural Scenic District, Sichuan, China. Journal of Hydro$\log y, 230,34-54$

MAGNIN F., 1997 - Travertinisation et paléoenvironnement du début de l'Holocène en France méditerranéenne : une succession malacologique dans les sédiments carbonatés de la vallée de la Tave (Laudin, Gard). Etudes de Géographie Physique, 26, 43-46.

MAGNIN F., GUENDON J.-L., VAUDOUR J., \& MARTIN P., 1991 - Les travertins : accumulations carbonatées associées aux systèmes karstiques, séquences sédimentaires et paléoenvironnements quaternaires. Bulletin de la Société Géologique de France, 162 (3), 585-594. 
MAGNIN F., \& THINON M., 1988 - Les travertins holocènes de Vauvenargues et de Saint Antonin (Bouches-du-Rhône) : nouvelles données sur les paléoenvironnements (malacologie, anthracologie). Les édifices travertineux et l'histoire de l'environnement dans le Midi de la France, U.A. 903 CNRS, Travaux 13, Aix en Provence, 63-72.

MAGNY M., LEUZINGER E., BORTENSCHLAGER S., \& HAAS J.-N., 2006 - Tripartite climate reversal in Central Europe 5600-5300 year ago. Quaternary Research, 65, 3-19.

MARTIN S., 2004 - Caractérisation de l'anthropisation à l'Holocène en Provence et en Languedoc oriental, par les mollusques terrestres. Thèse d'Archéologie, Paris I, 428 p.

MARTINEZ M., 2005 - Histoire biogéographique des pins de la section sylvestris (Pinus sylvestris, Pinus uncinata, Pinus mugo) dans les Alpes internes (Maurienne et Alpes italiennes) d'après l'analyse de macrorestes végétaux contenus dans les travertins. Master B.G.A.E., Université de Montpellier 2, 45 p.

MLAKAR J.M., DEGAUGUE F., LEROY S., GUENDON J.-L., \& AMBERT P., 1999 - Les travertins de la Guisane (col Lautaret, Hautes-Alpes, France) : caractères, datations et paléoenvironnement alpin holocène. Etudes de Géographie Physique, XXVII, Aix en Provence, 75-80.

NEVIERE C., 1996 - Les systèmes travertineux du Serre de Montdenier (Alpes de Haute-Provence). Etudes de Géographie Physique, 25, pp. 31-34

NICOL-PICHARD S., 1986 - Analyse pollinique de sédiments associés à des travertins, Jouques (Bouches-du-Rhône, France). Méditerranée, 1-2, 21-25.

OLLIVIER V., \& PINATEL A., 2000 - Etude de la dynamique érosive actuelle et holocène à l'échelle des ravines dans les terrains marneux des Alpes du Sud. Mémoire de Maîtrise de Géographie Physique, Université d'Aix-Marseille I, 114 p. + annexes.

OLLIVIER V., 2001 - Evolution géomorphologique postglaciaire du piémont méridional du grand Luberon en relation avec l'occupation humaine. Courrier Scientifique du parc naturel régional du Luberon, 5, 32-46.

OLLIVIER V., MIRAMONT C., \& MÜLLER A., 2004 - Le piémont méridional du Grand Luberon : De nouvelles données sur la morphogenèse postglaciaire en Basse Provence. Méditerranée, 1.2, 109-118.
OLLIVIER V., GUENDON J-L., MULLER A., \& MARTIN S., 2006 - Les travertins du Mirail (sud Luberon, Vaucluse), témoins des fluctuations morphosédimentaires holocènes. Actes de la Table Ronde en l'honneur de R. Neboit-Guilhot, "L'érosion entre société, climat et paléoenvironnement », Clermont-Ferrand, 25-27 mars 2004, Presses Universitaires Blaise Pascal, collection « Nature et Sociétés », (sous presse).

ROIRON P., ALI A.-A., GUENDON J.-L., MIGUERES M.-E., MULLER S., \& OLLIVIER V., 2006 - Paléodiversité de la végétation ligneuse enregistrée dans le travertin holocène du Serre de Montdenier (Alpes de Haute Provence, France). Quaternaire, ce volume.

VALERO-GARCES B.-L., ARENAS C., \& DELGADOHUERTAS A., 2001 - Depositional environments of Quaternary lacustrine travertines and stromatolites from high-altitude Andean lakes, northwestern Argentina. Canadian Journal of Earth Science, 38, 1263-1283.

VARELA F.-J., 1989 - Autonomie et connaissance : essai sur le vivant. Ed du Seuil, Paris, 247 p.

VAUDOUR J., 1986a - Travertins holocènes et pression anthropique. Méditerranée, 1-2, 168-172.

VAUDOUR J., 1986b - Introduction à l'étude des édifices travertineux holocènes. Méditerranée, 1-2, 3-10.

VAUDOUR J. (dir.), 1988 - Les édifices travertineux et l'histoire de l'environnement dans le Midi de la France. U.A. 903 CNRS, Travaux 13, Aix en Provence, $280 \mathrm{p}$

VAUDOUR J., 1994 - Evolution holocène des travertins de vallée dans le midi méditerranéen français. Géographie Physique et Quaternaire, 48 (3), 315-326.

VERNET J.L., \& VAUDOUR J. (dir), 1988-1990 - Milieux et anthropisation à l'Holocène en Méditerranée occidentale à partir des sites karstiques. Rapport final A.T.P. P.I.R.E.N., Montpellier-Aix en Provence, 215 p.

WALKER M.J.C., BJÖRCK S., LOWE J.J., CWYNAR L., JOHNSEN S., KNUDSEN K-L., WOHLFARTH B., \& INTIMATE GROUP, 1999 - Isotopic 'events' in the GRIP ice core : a stratotype for the Late Pleistocene. Quaternary Science Reviews, 18, 1143-1150. 